\title{
Algorithmic aspects in large deformation contact analysis using "Solid-Shell" elements
}

\author{
Matthias Harnau, Alexander Konyukhov, Karl Schweizerhof \\ Universität Karlsruhe, Institut für Mechanik
}

Institut für Mechanik

Kaiserstr. 12, Geb. 20.30

76128 Karlsruhe

Tel.: +49 (0) 721/ 608-2071

Fax: +49 (0) 721/ 608-7990

E-Mail: ifm@uni-karlsruhe.de

www.ifm.uni-karlsruhe.de 


\title{
Algorithmic aspects in large deformation contact analysis using 'Solid-Shell' elements
}

\author{
M. Harnau, A. Konyukhov and K. Schweizerhof
}

2004

\begin{abstract}
A special application for the so-called 'Solid-Shell' elements are sheet metal forming problems with high stretching and large local contact pressure where standard 2D-shells fail to converge resp. do not give reasonable results. To describe such kind of problems besides a full 3D continuum discretization appropriate contact formulations are necessary to introduce the contact condition of the metal sheets against the rigid tools. In this contribution a velocity description is taken for formulation of contact conditions. A penalty as well as an Augmented Lagrangian approach for frictionless contact is used as a first step in our developments. Special attention is paid to different numerical integration schemes of the contact integral and tangent matrices. As a result a series of different contact elements including various cases as "node-to-surface", "segment-to-segment" and "analytical rigid surface-to-segment" is considered under the unified description. For selected numerical examples the influence of the order of the quadrature formulae in a subdomain integration approach as well as the order of finite element interpolation used in the computations is discussed. The algorithms appear also to work well for friction type problems, which will be tested in a following paper.
\end{abstract}

Keywords Higher Order 'Solid-Shell' Elements, Large Deformations, Penalty Method, Augmented Lagrangian Method, Internal Geometry of the Contact Surfaces, Contact Problems, Sheet Metal Forming

\section{Introduction}

The so-called 'Solid-Shell' formulation as described in [10] and e.g. [19], [17] is based solely on displacement degrees of freedom belonging to the upper and lower shell surfaces and thus the use of rotational degrees of freedom can be avoided. As no kinematical assumption is applied beyond standard 3D continuum theory also general threedimensional material laws can be provided. In particular shell type problems with high stresses in thickness direction and considerable thinning due to stretching can be analyzed without further assumptions. Furthermore, to achieve a better geometric approximation beyond 'Solid-Shell' elements with bilinear in-plane shape functions also 
biquadratic in-plane shape functions are considered. To overcome the locking problems, which appear for both orders of interpolation, different schemes are used and finally as proposed for example in [9] almost locking free element formulations can be derived.

The main part of the paper focuses on large deformations contact problems. With standard nodal contact formulations the problem of weighting of the single nodal contribution occurs. As it is well known this is an effect of the under-integration of the contact integral and the corresponding tangent matrix. To overcome this problem a series of surface contact elements is investigated. These elements inherit the geometry of the surfaces of the 'Solid-Shell' elements. Therefore, instead of evaluating the contact conditions at the nodal points and determining the nodal forces directly, the contact forces are integrated numerically over the element area of surface contact elements as suggested in [15] and [24]. An improvement appears possible by using an integration in the subdomains of the contact element. A velocity description [14] is used for the formulation of the contact conditions and for the derivation of the contact tangent matrix as well. Both, penalty and Augmented Lagrangian methods are used to enforce the contact conditions.

The contact forces are discontinuous over the contact surface and, therefore, the numerical integration gives a better result, if the number of integration points is increased. For further investigations a series of contact elements including a different approach for the evaluation of the contact integral is considered to check the effect of the numerical integration more closely. Contact between rigid analytically defined surfaces and deformable bodies is separately treated. Finally, after demonstrating the effect of various integration schemes on some simple examples, a sheet metal forming example with rather industrial content is taken to demonstrate various characteristics within this process.

\section{The 'Solid-Shell' concept}

In this section the basic features of the 'Solid-Shell' concept are briefly reviewed. For a detailed explanation we refer to [10] resp. [19] and [17] for similar elements. Two different types of 'Solid-Shells' have been developed, see fig. 1, the bilinear element type with four nodes on the upper and lower shell surface each and the biquadratic element type with nine in-plane nodes on each surface. From the discrete nodal coordinates and displacements the geometry as well as the displacement field is approximated using the bilinear resp. biquadratic Lagrange shape functions for in-plane approximation and a linear interpolation in thickness direction.

As is well known the 'Solid-Shell' elements suffer from many locking effects. To avoid locking, methods of underintegrating the volume integrals (SRI [22]), an interpolation of transverse shear strains, resp. membrane strains and even thickness strains (ANS- 

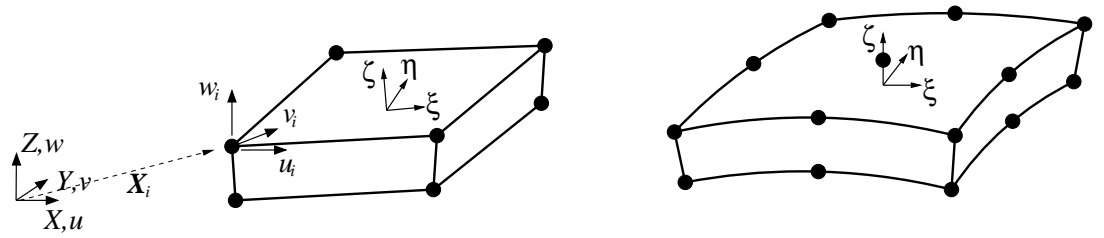

Figure 1: Solid-Shell' elements with bilinear and biquadratic shapes

method [2], [4], [3]) as well as mixed formulations (EAS-method [20]) are applicable. A complete discussion about these locking phenomena and different developed element versions is given e.g. in [9]. Finally, almost locking free 3D-shell elements are avail-

\begin{tabular}{|l|c|c|c|c|}
\hline Elementname & $\begin{array}{c}\text { in-plane } \\
\text { approximation }\end{array}$ & $\begin{array}{c}\text { membrane } \\
\text { strain } \\
\text { modification }\end{array}$ & $\begin{array}{c}\text { thickness } \\
\text { strain } \\
\text { modification }\end{array}$ & $\begin{array}{c}\text { transverse } \\
\text { shear strain } \\
\text { modification }\end{array}$ \\
\hline ANS3DEAS & bilinear Lagr. & - & EAS & ANS \\
\hline EAS3DEAS & bilinear Lagr. & EAS & EAS & ANS \\
\hline MI9K3DEAS & biquadratic Lagr. & ANS & EAS & ANS \\
\hline
\end{tabular}

Table 1: Nomenclature for 'Solid-Shell' element formulations

able. The nomenclature for the element versions relevant for the investigations in the following chapters is given in tab. 1.

The use of nonlinear material laws, necessary for the treatment of large deformation problems, is described in detail e.g. in [11]. It should be mentioned that in contrast to the degenerated shell concept strains and stresses in thickness direction are included in the 'Solid-Shell' concept. Thus general three-dimensional material laws can be used without any modification and general 3D stress and strain states can be treated directly.

\section{Different contact algorithms in frictionless con- tact analysis}

\subsection{Velocity description}

A specific derivation based on a velocity description [14] can be applied to describe contact conditions with any type of curved surfaces. For this purpose, the following tensor notations have to be introduced with surface coordinates $\xi, \eta$ and the normal 
coordinate $\zeta$ respectively

$$
\xi^{1}=\xi, \quad \xi^{2}=\eta, \quad \xi^{3}=\zeta
$$

The local surface coordinate system is defined by means of surface tangent vectors:

$$
\boldsymbol{x}_{1}=\frac{\partial \boldsymbol{x}}{\partial \xi^{1}}=\overline{\boldsymbol{x}}_{, \xi}, \quad \boldsymbol{x}_{2}=\frac{\partial \boldsymbol{x}}{\partial \xi^{2}}=\overline{\boldsymbol{x}}_{, \eta}
$$

and a normal surface vector

$$
\mathbf{n}=\frac{\boldsymbol{x}_{1} \times \boldsymbol{x}_{2}}{\left|\boldsymbol{x}_{1} \times \boldsymbol{x}_{2}\right|}
$$

where $\overline{\boldsymbol{x}}=\sum_{k=1}^{n} N_{k}\left(\xi^{1}, \xi^{2}\right) \overline{\boldsymbol{x}}_{e}^{(k)}$ is the parameterization of the surface inherited from the finite element discretization.

We then introduce a local coordinate system as

$$
\mathbf{r}_{s}\left(\xi^{1}, \xi^{2}, \xi^{3}\right)=\overline{\boldsymbol{x}}\left(\xi^{1}, \xi^{2}\right)+\mathbf{n} \xi^{3}
$$

where $\mathbf{r}_{s}$ is a "slave" point and $\overline{\boldsymbol{x}}$ is its projection on the surface. Within the "masterslave" approach a point of one body which penetrates into another is called "slave" point. Additional specifications of the "slave" point will be discussed further when the integration procedure is shown in more detail. For the coordinate system defined in eqn. (4) it is assumed that the well known closest point projection procedure [16], [24] has been already fulfilled. Therefore, the third local coordinate $\xi^{3}$ is the value of a penetration

$$
\xi^{3}=g_{N}=\left(\mathbf{r}_{s}-\overline{\boldsymbol{x}}\right) \cdot \mathbf{n}
$$

The full time derivative of eqn. (4) gives

$$
\mathbf{v}_{s}=\mathbf{v}+\xi^{3} \frac{\partial \mathbf{n}}{\partial t}+\mathbf{n} \dot{\xi}^{3}+\left(\overline{\boldsymbol{x}}-\xi^{3} h_{j}^{i} \boldsymbol{x}_{i}\right) \dot{\xi}^{j}
$$

where $\mathbf{v}_{s}=\frac{d}{d t} \mathbf{r}_{s}\left(t, \xi^{1}, \xi^{2}, \xi^{3}\right)$ is the velocity of the "slave" point, $\mathbf{v}=\frac{\partial \overline{\boldsymbol{x}}}{\partial t}$ is the translation velocity of the projection of the "slave" point, and $h_{j}^{i}$ are the mixed components of the curvature tensor. 
A dot product eqn. (6) with the surface tangent vectors eqn. (2) gives after some algebraic transformation the convective velocity components in the following form

$$
\dot{\xi}^{j}=\hat{a}^{i j}\left[\left(\mathbf{v}_{s}-\mathbf{v}\right) \cdot \boldsymbol{x}_{i}-\xi^{3}\left(\frac{\partial \mathbf{n}}{\partial t} \cdot \boldsymbol{x}_{i}\right)\right], \quad i, j=1,2
$$

where $\hat{a}^{i j}$ are the contravariant components of $\left(a_{i j}-\xi^{3} h_{i j}\right)$, and $a_{i j}$ are the covariant components of the metric tensor. A dot product of the relative velocity eqn. (6) with the normal (3) gives a time derivative of the penetration

$$
\dot{\xi}^{3}=\left(\mathbf{v}_{s}-\mathbf{v}\right) \cdot \mathbf{n}
$$

As shown in [14], the following reduction of eqn. (7)

$$
\dot{\xi}^{j}=a^{i j}\left(\mathbf{v}_{s}-\mathbf{v}\right) \cdot \boldsymbol{x}_{i}
$$

gives a first order description with respect to the penetration $g_{N}=\xi^{3}$, which is consistent with the contact integral. The contact integral has then the following form

$$
\begin{aligned}
\delta W_{c} & =\int_{s} N \delta g_{N} d s+\int_{s} T_{j} \delta \xi^{j} d s= \\
& =\int_{s} N\left(\delta \mathbf{r}_{s}-\delta \boldsymbol{x}\right) \cdot \mathbf{n} d s+\int_{s} T^{j}\left(\delta \mathbf{r}_{s}-\delta \boldsymbol{x}\right) \cdot \boldsymbol{x}_{i} d s
\end{aligned}
$$

where the full contact tractions $\mathbf{T}$ are expressed as a sum of tangential and normal forces

$$
\mathbf{T}=N \mathbf{n}+T^{i} \boldsymbol{x}_{i}
$$

The contact integral in eqn. (10) is computed over the slave surface.

\section{Tangent matrix for the non-frictional case with a penalty formulation}

In the non-frictional case, i.e. $N \neq 0$ and $T^{j}=0$, the penalty formulation of the contact condition leads to the following contact functional:

$$
\delta W_{c}^{N}=\int_{s} \epsilon_{p}\left\langle g_{N}\right\rangle \delta g_{N} d s
$$

with the penalty parameter $\epsilon_{p}$, and the Macauley brackets \langle\rangle in the form

$$
\left\langle g_{N}\left(\xi^{1}, \xi^{2}\right)\right\rangle=\left\{\begin{array}{c}
0, \text { if } g_{N}\left(\xi^{1}, \xi^{2}\right)>0 \\
g_{N}, \text { if } g_{N} \leq 0
\end{array}\right.
$$


If the equation for the convective velocities (9) is taken into account the linearized contact integral (12) has the following form:

$$
\begin{aligned}
& D_{v}\left(\delta W_{c}^{N}\right)= \\
& =\int_{S} \epsilon_{p} H\left(-g_{N}\right)\left(\delta \mathbf{r}_{s}-\delta \boldsymbol{x}\right) \cdot(\mathbf{n} \otimes \mathbf{n})\left(\mathbf{v}_{s}-\mathbf{v}\right) d S \\
& -\int_{S} \epsilon_{p} H\left(-g_{N}\right) g_{N}\left(\delta \boldsymbol{x}_{, j} \cdot a^{i j}\left(\mathbf{n} \otimes \boldsymbol{x}_{i}\right)\left(\mathbf{v}_{s}-\mathbf{v}\right)+\left(\delta \mathbf{r}_{s}-\delta \boldsymbol{x}\right) \cdot a^{i j}\left(\boldsymbol{x}_{j} \otimes \mathbf{n}\right) \mathbf{v}_{, i}\right) d S(15) \\
& -\int_{S} \epsilon_{p} H\left(-g_{N}\right) g_{N}\left(\delta \mathbf{r}_{s}-\delta \boldsymbol{x}\right) \cdot h^{i j}\left(\boldsymbol{x}_{i} \otimes \boldsymbol{x}_{j}\right)\left(\mathbf{v}_{s}-\mathbf{v}\right) d S
\end{aligned}
$$

where $H\left(-g_{N}\right)$ is the Heaviside function denoting that the integral is computed, if the penetration is nonpositive.

The resulting normal contact matrix is naturally subdivided into a main matrix eqn. (14), a "rotational" matrix (15) and a "curvature" matrix (16). For details about the derivation of the matrices as well as the influence of each part on the convergence of the nonlinear solution in the case of different approximations of contact surfaces we refer to [14].

\subsection{Augmented Lagrangian contact formulation}

As an alternative approach to achieve satisfaction of the contact condition the method of Lagrangian multipliers could be used. The Lagrangian multipliers allow in general to fulfill any constraint condition exactly, but then the system of equations is extended by additional unknowns $\lambda$. Another disadvantage is that the linearization of the Lagrangian term leads to zero entries on the main diagonal of the stiffness matrix; even the size of the equation system is changing in the solution process, because the multipliers vanish in the case of no contact resp. penetration. This all leads often to numerical problems and particularly enlarged effort in the solution process; further special solution techniques would be required to overcome the problem with zero diagonal entries.

Therefore besides the penalty method introduced above the so called Augmented Lagrangian approach as proposed in [25] and [21] is also tested for the contact description. This method can be considered as a combination of the penalty method and the method of Lagrangian multipliers. The standard functional is then extended by two additional parts:

$$
\Pi_{A}=\int_{A_{c}} \lambda\left\langle g_{N}\right\rangle d A_{c}+\frac{1}{2} \int_{A_{c}} \varepsilon_{p}\left\langle g_{N}\right\rangle^{2} d A_{c},
$$


where again the gap function $g_{N}\left(\xi^{1}, \xi^{2}\right)$ is reformulated as a penetration function according to eqn. (13). In order to simplify the solution the Lagrangian parameters $\lambda$, here the contact force/stress, are supposed to be fixed within an iteration in the Newton-Raphson solution. Therefore they do not have to be taken into account as additional variables for the variation and linearization of the functional. When the equilibrium iteration process is finished, the constraint condition is checked, and, if necessary, the Lagrangian parameters are updated resp. augmented by

$$
\lambda=\lambda+\varepsilon_{p}\left\langle g_{N}\right\rangle,
$$

and an additional equilibrium iteration process is started with the increased contact force; (the complete algorithm which is known as Uzawa algorithm [16] is summarized in tab. 2). Turning off the augmentation procedure this approach is identical to a pure

\section{Load step $n$}

1) Initialize Augmented Lagrangian Parameters $\boldsymbol{\lambda}_{(k)}^{(n)}=\boldsymbol{\lambda}_{(k)}^{(n-1)}$ and $\boldsymbol{\lambda}_{(0)}^{(0)}=0$

2) Initialize Augmented Lagrangian iteration counter $k=0$

3) Solve nonlinear equation system for $\boldsymbol{d}$ using Newton-Raphson iteration scheme $\left(\boldsymbol{\lambda}_{(k)}^{(n)}\right.$ is fixed $)$

4) Check penetration of nodal resp. integration points to find maximal penetration $\max g_{N i}$ at point $i$ if $\max g_{N i} \leq$ tol $g_{N}$ next load step $n=n+1$, goto 1 ) else $\boldsymbol{\lambda}_{(k+1)}^{(n)}=\boldsymbol{\lambda}_{(k)}^{(n)}+\varepsilon_{p} \boldsymbol{g}_{\boldsymbol{N}}, k=k+1$, goto 3$)$

Table 2: Nested iteration scheme for Augmented Lagrangian approach

penalty formulation.

\subsection{Computation of the contact integral}

Within the finite element method the contact integral in eqn. (10), leading to the residual, as well as the integrals in equations (14), (15), (16), leading to the consistent tangent matrix, have to be computed using one or another quadrature formulae. In the most common approach known as "node-to-surface" technique the value at the nodes from the finite element discretization of the "slave" part is taken directly. As is well 
known, this technique can be only directly applied in the case of linear approximations for both "slave" and "master" parts, see [26], and it does not satisfy the patch test, see [6]. This fact can be explained as under-integration of the contact integral, because the Lobbatto quadrature formula with only two integration points gives exactly a nodal collocation formula in this case. As an improvement different quadrature formulae of higher order can be used. In this situation the question arrises: How many integration points have to be taken in order to achieve a certain error bound? The usual formula to estimate the integration error does not give the correct answer, because it requires differentiability of the integrand up to the certain order. This is not the case for the computation of the contact integral, which is discussed in the following: the function in the integral is defined on the master element, but the computation of the integral has to be done over the unknown slave surface. In practice, the penetration of "slave" points, e.g. integration points, from different "slave" segments into the master segment is checked, see fig. 4. This can be considered as integration of auxiliary functions over the known master surface which again define a function which is discontinuous on the master surface.

As a representative example to show that the problem of integration of discontinuous functions arises during the "master-slave" approach we consider the classical Hertz problem. Assume that the contact problem of a cylinder and a semi-infinite elastic plane, see fig. 2, is solved by the standard penalty approach for the finite element method. Let $C D$ be a $2 \mathrm{D}$ contact element. Controlling the process with an applied vertical displacement $h$, the cylinder penetrates into the plane within the first iteration as shown in fig. 2. A characteristic quantity for the satisfaction of contact is the value of the energy associated with the penalty form in e.g. the first iteration. It has the following form:

$$
E_{g}=\frac{1}{2} \int_{A B} \varepsilon_{N} g_{N}^{2} d x=\frac{\varepsilon_{N}}{2} J
$$

The value of the integral $J$ in eqn. (2) can be evaluated in closed form, because both the penetration $g_{N}$ and the contact zone $A B$ are defined from the specific geometry as:

$$
g_{N}=R-h-\sqrt{R^{2}-x^{2}}, \quad A B=2 \sqrt{h(2 R-h)} .
$$

The integral $J$ after evaluation and some transformations has the following form:

$$
\begin{aligned}
J & =\int_{-\sqrt{h(2 R-h)}}^{+\sqrt{h(2 R-h)}}\left(R-h-\sqrt{R^{2}-x^{2}}\right)^{2} d x= \\
& =\frac{2}{3}\left(3 R^{2}-2 R h+h^{2}\right) \sqrt{h(2 R-h)}-2 R^{2}(R-h) \arcsin \frac{\sqrt{h(2 R-h)}}{R} .
\end{aligned}
$$




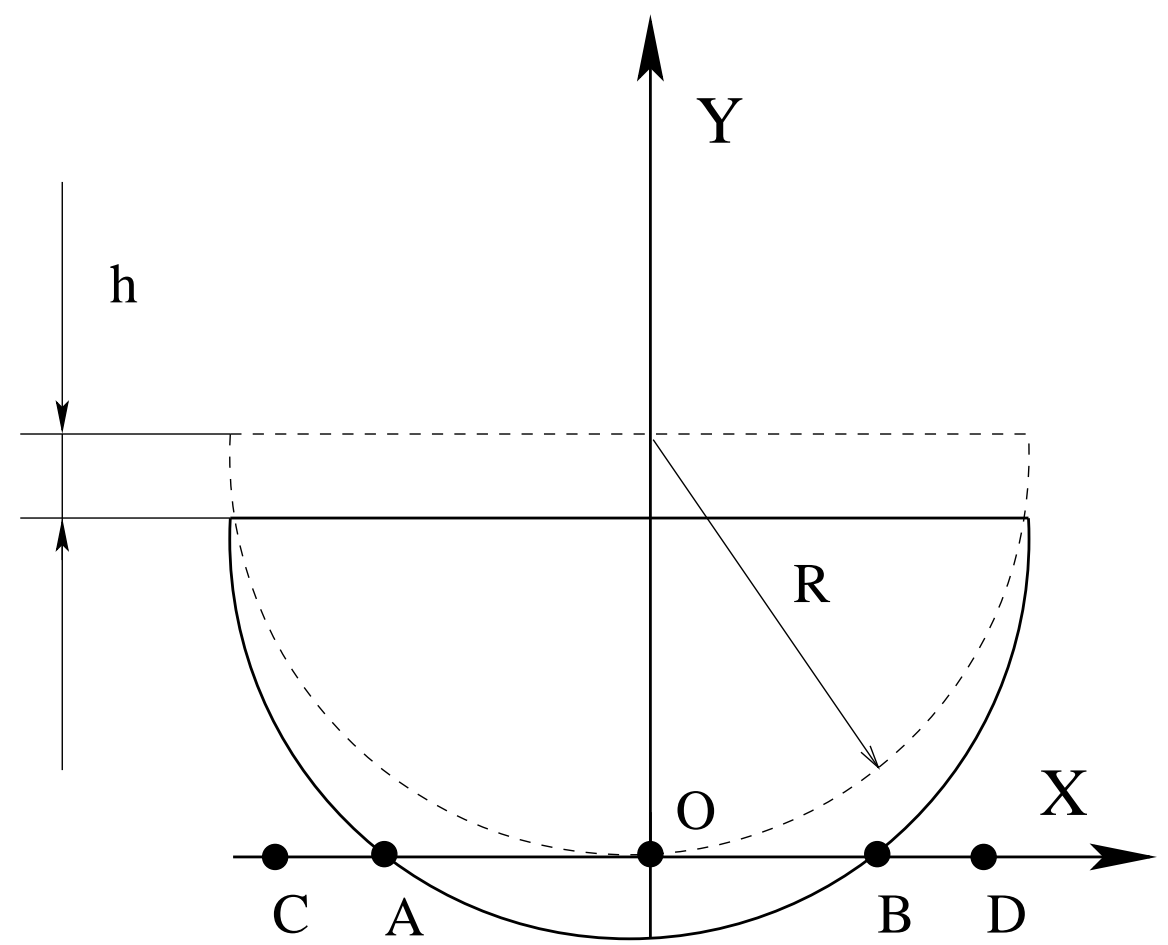

Figure 2: Cylinder during the first iteration

Within the finite element solution the value of the integral $J$ becomes:

$$
J=\int_{A C}\left\langle g_{N}\right\rangle^{2} d x
$$

where \langle\rangle denotes the Macauley brackets see eqn. (13). The value of the integral is computed over the contact element $C D$, while the contact region $A B$ is detected via the integration points, which then leads in general to a discontinuous function defined over the contact element $C D$. Before comparing the results, we describe in the following section one of the techniques to integrate discontinuous functions [7].

\section{Integration schemes using subdivision into subdomains}

The a-priori error estimation in the case of the application of Gauss quadrature rules for discontinuous functions is a more complicated question, because it is necessary to know the behavior of the integrand, see e.g. [7]. However, this is in general not known in the considered cases of rather general contact surfaces. One can only expect, that increasing the number of integration points leads to a reduction of the integration error. As an improved and efficient technique to decrease the integration error a subdivision of the integration area into subdomains together with lower order integration in each 
subdomain can be used (see e.g. [7]). With the same number of integration points as for a standard Gauss integration this technique leads to a smaller integration error, as is shown in the following.

Let $A$ be an area of element. In the case of a quadrilateral contact element with area $A$, this can be subdivided into non-overlapping subdomains $A_{i j}$ :

$$
A=\bigcup_{i j} A_{i j}
$$

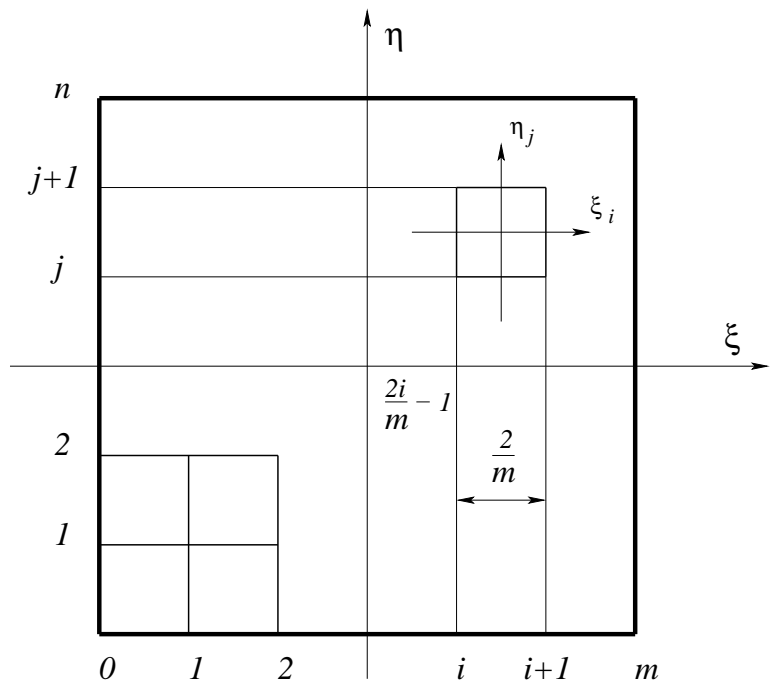

Figure 3: Subdivision of the contact segment into subdomains for integration

Now we consider the subdivision of $A$ with the local coordinate system $\xi, \eta$ into rectangular subdomains, see fig. 3, e.g. into $m$ parts along the $\xi$ axis and into $n$ parts along $\eta$ axis. In each subdomain a separate local coordinate system $\xi_{i}, \eta_{j}$, which has to satisfy the following conditions, is introduced:

$$
\xi_{i}=-1 \text { if } \xi=\frac{2 i}{m}-1, \quad \xi_{i}=1 \text { if } \xi=\frac{2(i+1)}{m}-1, \quad i=0,1,2, \ldots m-1
$$

and

$$
\eta_{j}=-1 \text { if } \eta=\frac{2 j}{n}-1, \quad \eta_{j}=1 \text { if } \eta=\frac{2(j+1)}{n}-1, \quad j=0,1,2, \ldots n-1
$$

Then, the transformation of the coordinates $\xi_{i}, \eta_{j}$ into $\xi, \eta$ can be written as:

$$
\xi=\frac{\xi_{i}}{m}+\frac{2 i+1}{m}-1, \quad \eta=\frac{\eta_{j}}{n}+\frac{2 j+1}{n}-1 .
$$


Finally, the integration of a function $f(\xi, \eta)$ over the area $A$ in the local coordinate system $\xi, \eta$ leads to a sum of integrals over each subdomain $A_{\xi_{i}, \eta_{j}}$ :

$$
\begin{aligned}
& \int_{-1}^{+1} \int_{-1}^{+1} f(\xi, \eta) d \xi d \eta=\sum_{i=0}^{m-1} \sum_{j=0}^{n-1} \int_{A_{\xi_{i}, \eta_{j}}} f(\xi, \eta) d \xi d \eta= \\
& \quad=\frac{1}{m \cdot n} \sum_{i=0}^{m-1} \sum_{j=0}^{n-1} \int_{-1}^{+1} \int_{-1}^{+1} f\left(\frac{\xi_{i}}{m}+\frac{2 i+1}{m}-1, \frac{\eta_{j}}{n}+\frac{2 j+1}{n}-1\right) d \xi_{i} d \eta_{j}
\end{aligned}
$$

Each integral in formula (27) is computed via standard quadrature formulae, e.g. Gauss integration.

In order to consider general features of the proposed approach, first the exact value of the integral $J$ in eqn. (21) is compared with the computed value in (22) for a line contact. The following parameters are taken: radius of cylinder $R=2.0$, vertical displacement $h=0.1$, length of element $C D=2.0$. In this case as exact value of the integral $J=6.7099 \cdot 10^{-3}$ and as length of the contact zone $A B=1.249$ are obtained. The following relative error $e$ is used for comparison of the computed value $J_{\text {com }}$ with the exact value $J$ :

$$
e=\frac{J_{\text {exact }}-J_{\text {com }}}{J_{\text {exact }}} \cdot 100 \%
$$

Tab. 3 shows the relative error $e$ in the case of various numbers of Gauss points and subdivisions.

As expected, the formula with subdivisions leads to a smaller error than the standard single domain Gauss formula. It is obvious that among the formulae with a fixed total number of integration points the smallest error is obtained by the formula that combines both the maximum number of subdivisions and the maximum number of Gauss points which can be independently chosen.

After the description of the contact elements in the following part we will show that the proposed approach allows first to diminish the error for the patch test and second to improve the quality of the results, e.g. the load-displacement curve.

\section{Finite element discretization of contact}

Several constructions of a contact element resp. contact segment depending on the chosen integration scheme are considered. The well known "node-to-surface" element with nodal collocation, a "segment-to-segment" element with an auxiliary integration as well as a contact element for rigid surfaces defined by analytical functions are generalized by the development of a specific position matrix. 


\begin{tabular}{|c|c|c|}
\hline No. of Gauss points & No. of subdivisions & $e \%$ \\
\hline 2 & 1 & 93.4232 \\
\hline 3 & 1 & -32.4737 \\
\hline 5 & 1 & 5.4618 \\
\hline 6 & 1 & -2.4670 \\
\hline 3 & 2 & 4.7420 \\
\hline 2 & 3 & -0.1113 \\
\hline 7 & 1 & -1.0916 \\
\hline 10 & 1 & -0.6844 \\
\hline 5 & 2 & 0.2399 \\
\hline 2 & 5 & 0.2018 \\
\hline 20 & 1 & -0.0669 \\
\hline 10 & 2 & 0.1147 \\
\hline 5 & 4 & 0.0978 \\
\hline 4 & 5 & 0.0153 \\
\hline 2 & 10 & 0.0271 \\
\hline 40 & 1 & 0.0114 \\
\hline 20 & 2 & 0.0196 \\
\hline 10 & 4 & -0.0137 \\
\hline 8 & 5 & -0.0021 \\
\hline 5 & 8 & 0.0001 \\
\hline 4 & 10 & 0.0029 \\
\hline 2 & 20 & 0.0142 \\
\hline
\end{tabular}

Table 3: Relative error in energy of contact integral for penalty formulation; comparing standard Gauss integration with subdivisional Gauss integration in subdomains

\subsection{Node-to-surface contact element}

First, a "node-to-surface" approach is considered. In this case each "slave" node is treated separately. If the approximation of the 'master' surface is defined by $n$ nodes and the 'slave' node is the $(n+1)$ 'th node, then the nodal vector for the contact element can be written in the following form:

$$
\overline{\boldsymbol{x}}_{e}^{T}=\left\{x_{1}^{(1)}, x_{2}^{(1)}, x_{3}^{(1)}, x_{1}^{(2)}, x_{2}^{(2)}, x_{3}^{(2)}, \ldots, \ldots, x_{1}^{(n)}, x_{2}^{(n)}, x_{3}^{(n)}, x_{1}^{(n+1)}, x_{2}^{(n+1)}, x_{3}^{(n+1)}\right\}^{T} \cdot(29)
$$


Position matrices with dimension $3 \times(n+1)$ are necessary in order to define the nodal displacement

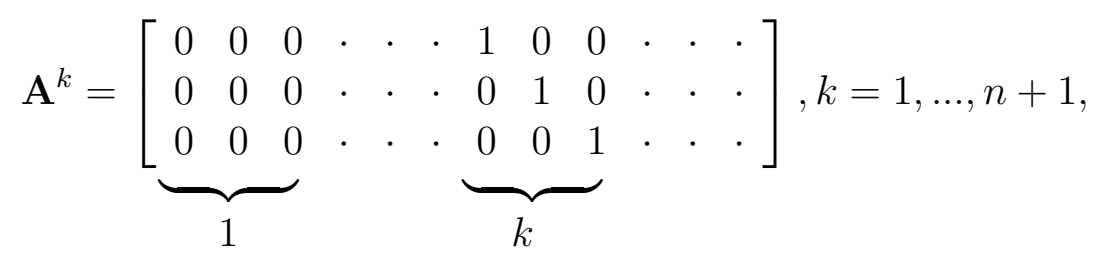

where the $3 \times 3$ unity matrix is placed here at position $k$. Then a position vector for the $k$-th node $\boldsymbol{x}^{(k)}=\left\{x_{1}^{(k)}, x_{2}^{(k)}, x_{3}^{(k)}\right\}$ in the global Cartesian reference frame can be written as

$$
\boldsymbol{x}^{(k)}=\mathbf{A}^{k} \overline{\boldsymbol{x}}_{e}, \quad k=1,2, \ldots, n
$$

and for the slave node $S$ as

$$
\mathbf{r}_{s}=\mathbf{A}^{(n+1)} \overline{\boldsymbol{x}}_{e}
$$

With $N_{(k)}\left(\xi^{1}, \xi^{2}\right), \quad k=1,2, \ldots, n$ as the shape functions for the master surface parameterization, the projection of the slave point $C$ on the master surface is defined as

$$
\overline{\boldsymbol{x}}=\sum_{k=1}^{n} N_{(k)}\left(\xi_{c}^{1}, \xi_{c}^{2}\right) \mathbf{A}^{k} \overline{\boldsymbol{x}}_{e}=\mathbf{A}^{c} \overline{\boldsymbol{x}}_{e}
$$

with

$$
\mathbf{A}^{c}=\sum_{k=1}^{n} N_{(k)}\left(\xi_{c}^{1}, \xi_{c}^{2}\right) \mathbf{A}^{k}
$$

On the basis of this notation, the vector $\delta \mathbf{r}_{s}-\delta \overline{\boldsymbol{x}}$ has the following form

$$
\delta \mathbf{r}_{s}-\delta \overline{\boldsymbol{x}}=\left(\mathbf{A}^{(n+1)}-\mathbf{A}^{c}\right) \delta \overline{\boldsymbol{x}}_{e}=\mathbf{A} \delta \overline{\boldsymbol{x}}_{e}
$$

where a new matrix $\mathbf{A}=\mathbf{A}^{(n+1)}-\mathbf{A}^{c}$ has been defined as

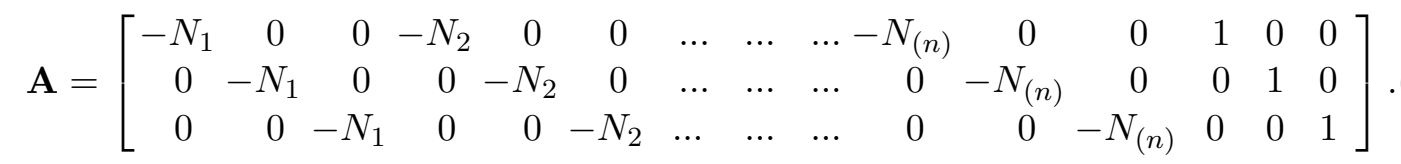


The relative velocity vector $\left(\mathbf{v}_{s}-\mathbf{v}\right)$ has the identical structure:

$$
\mathbf{v}_{s}-\mathbf{v}=\left(\mathbf{A}^{(n+1)}-\mathbf{A}^{c}\right) \mathbf{v}_{e}=\mathbf{A} \mathbf{v}_{e}
$$

For further developments only the matrix of the shape function derivatives $\mathbf{A}_{, j}, i=1,2$ has to be specified

$$
\mathbf{A}_{, j}=\left[\begin{array}{ccccccccccccccc}
N_{1, j} & 0 & 0 & N_{2, j} & 0 & 0 & \ldots & \ldots & \ldots & N_{(n), j} & 0 & 0 & 0 & 0 & 0 \\
0 & N_{1, j} & 0 & 0 & N_{2, j} & 0 & \ldots & \ldots & \ldots & 0 & N_{(n), j} & 0 & 0 & 0 & 0 \\
0 & 0 & N_{1, j} & 0 & 0 & N_{2, j} & \ldots & \ldots & \ldots & 0 & 0 & N_{(n), j} & 0 & 0 & 0
\end{array}\right],
$$

in order to describe the vectors $\delta \boldsymbol{x}_{, j}$ and $\mathbf{v}_{, j}$

$$
\delta \boldsymbol{x}_{, j}=\mathbf{A}_{, j} \overline{\boldsymbol{x}}_{e}, \quad \mathbf{v}_{, j}=\mathbf{A}_{, j} \mathbf{v}_{e} .
$$

\subsection{Segment-to-segment contact element}

For the auxiliary integration rule we have to specify the number and location of the integration points on the slave elements. Therefore, both a "master" and a "slave" surface have to be specified for a contact segment.

Let ABDE be a master segment (see fig. 4) defined by $n$ nodes

$$
\left\{x_{1}^{(1)}, x_{2}^{(1)}, x_{3}^{(1)}, x_{1}^{(2)}, x_{2}^{(2)}, x_{3}^{(2)}, \ldots, x_{1}^{(n)}, x_{2}^{(n)}, x_{3}^{(n)}\right\}^{T},
$$

and abde be a slave segment defined by $m$ nodes

$$
\left\{y_{1}^{(1)}, y_{2}^{(1)}, y_{3}^{(1)}, y_{1}^{(2)}, y_{2}^{(2)}, y_{3}^{(2)}, \ldots, y_{1}^{(m)}, y_{2}^{(m)}, y_{3}^{(m)}\right\}^{T} \text {. }
$$

The shape functions $N_{k}\left(\xi_{N}^{1}, \xi_{N}^{2}\right), k=1,2, \ldots n$ and $M_{k}\left(\xi_{M}^{1}, \xi_{M}^{2}\right), k=1,2, \ldots m$ are defined for the "master" and the "slave" segment respectively. A displacement vector for the contact segment is defined by $n+m$ nodes as

$$
\begin{aligned}
\mathbf{u}^{T} & =\left\{u_{1}^{(1)}, u_{2}^{(1)}, u_{3}^{(1)}, u_{1}^{(2)}, u_{2}^{(2)}, u_{3}^{(2)}, \ldots, u_{1}^{(n)}, u_{2}^{(n)}, u_{3}^{(n)}\right. \\
& \left.u_{1}^{(n+1)}, u_{2}^{(n+1)}, u_{3}^{(n+1)}, u_{1}^{(n+2)}, u_{2}^{(n+2)}, u_{3}^{(n+2)}, \ldots, u_{1}^{(n+m)}, u_{2}^{(n+m)}, u_{3}^{(n+m)}\right\}^{T} .
\end{aligned}
$$

The position matrices $\mathbf{A}^{k}$ (see eqn. 30), which now have $3 \times(n+m)$ dimension, define, similar to eqn. (35), the following matrix $\mathbf{A}$

$$
\begin{aligned}
& \mathbf{A}=\left[\begin{array}{cccccccccc}
-N_{1} & 0 & 0 & -N_{2} & 0 & 0 & \ldots & -N_{(n)} & 0 & 0 \\
0 & -N_{1} & 0 & 0 & -N_{2} & 0 & \ldots & 0 & -N_{(n)} & 0 \\
0 & 0 & -N_{1} & 0 & 0 & -N_{2} & \ldots & 0 & 0 & -N_{(n)}
\end{array}\right. \\
& \left.\begin{array}{cccccccccc}
M_{1} & 0 & 0 & M_{2} & 0 & 0 & \ldots & -M_{(m)} & 0 & 0 \\
0 & M_{1} & 0 & 0 & M_{2} & 0 & \ldots & 0 & -M_{(m)} & 0 \\
0 & 0 & M_{1} & 0 & 0 & M_{2} & \ldots & 0 & 0 & -M_{(m)}
\end{array}\right] \text {. }
\end{aligned}
$$




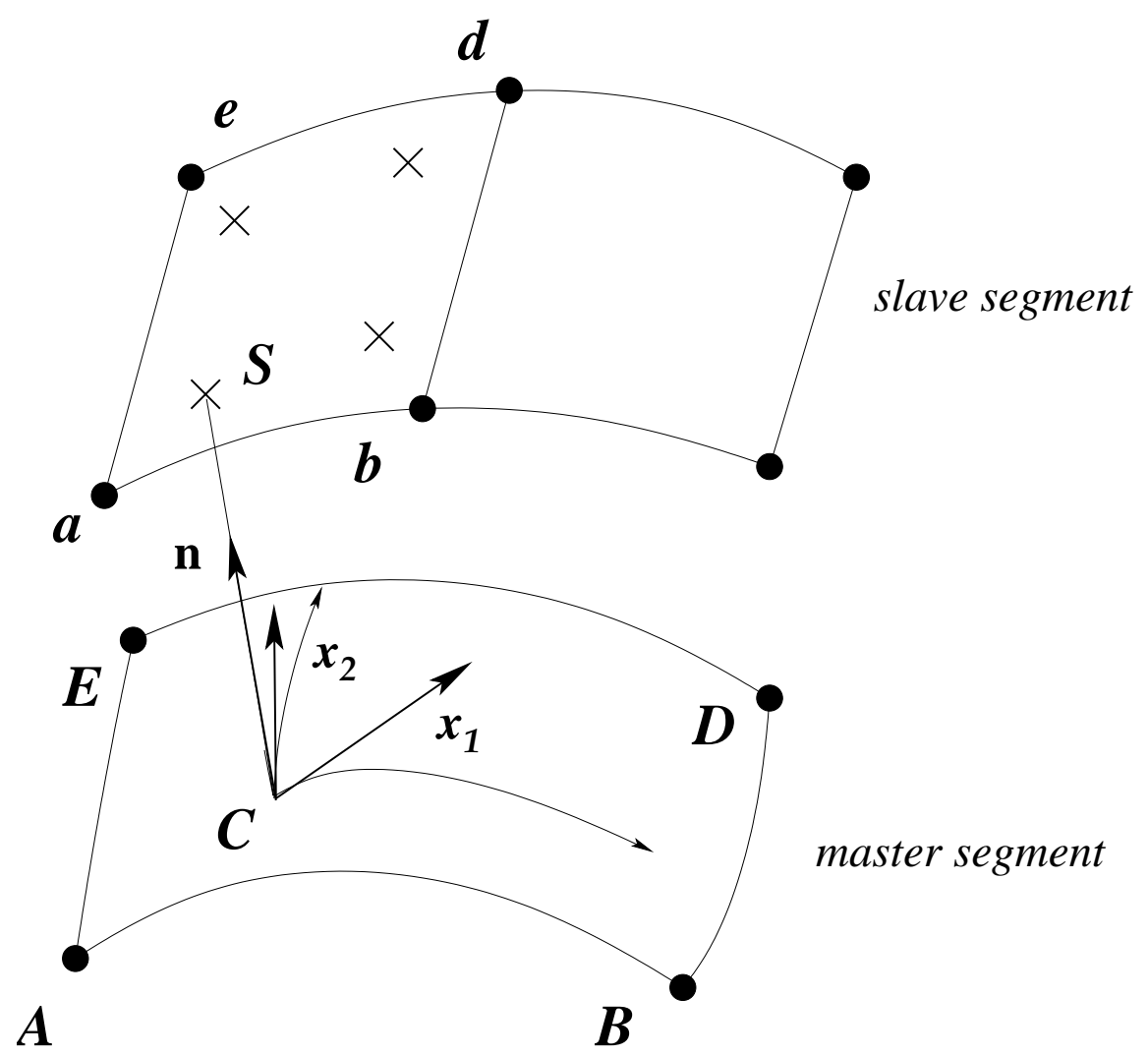

Figure 4: Segment-to-segment contact segment

A finite element approximation for the projection point $\overline{\boldsymbol{x}}$ is defined as

$$
\overline{\boldsymbol{x}}=\sum_{k=1}^{n} N_{k}\left(\xi_{N}^{1}, \xi_{N}^{2}\right) \mathbf{A}^{k} \overline{\boldsymbol{x}}_{e}
$$

and for "slave" points as

$$
\left.\mathbf{r}_{s}\right|_{\xi_{M}^{1}=\xi_{I}^{1}, \quad \xi_{M}^{2}=\xi_{J}^{2}}=\sum_{k=1}^{m} M_{k}\left(\xi_{I}^{1}, \xi_{J}^{2}\right) \mathbf{A}^{(k+n)} \overline{\boldsymbol{x}}_{e}
$$

where $\xi_{1}^{I}, \xi_{2}^{J}$ are integration points.

Since the differential operations in the tangent matrix, see eqns. (14), (15) and (16), are defined only on the master surface, the structure of the matrix of the shape function derivatives in eqn. (38) remains the same, namely

$$
\mathbf{A}_{, j}=\left[\begin{array}{ccccccc}
-N_{1, j} & 0 & 0 & -N_{2, j} & 0 & 0 & \cdots \\
0 & -N_{1, j} & 0 & 0 & -N_{2, j} & 0 & \cdots \\
0 & 0 & -N_{1, j} & 0 & 0 & -N_{2, j} & \cdots
\end{array}\right.
$$




$$
\left.\begin{array}{cccccccccc}
-N_{(n), j} & 0 & 0 & 0 & 0 & 0 & \ldots & 0 & 0 & 0 \\
0 & -N_{(n), j} & 0 & 0 & 0 & 0 & \ldots & 0 & 0 & 0 \\
0 & 0 & -N_{(n), j} & 0 & 0 & 0 & \ldots & 0 & 0 & 0
\end{array}\right]
$$

Based on the matrices defined in eqn. (41) and (44), the necessary expressions for the contact formulation result in the same form, see eqns. (35), (37) and (39).

Remark The "segment-to-segment" contact segment is constructed independently of the chosen quadrature formula. It should be noticed that the Lobatto quadrature formula (see e.g. [7] for a further discussion about numerical integration) with only $2 \times 2$ integration points leads exactly to the result of the "node-to-surface" element.

\subsection{Contact with rigid surfaces described by analytical func- tions}

If a body contacts a rigid surface, the latter one is chosen as a "slave" surface in our description, but the integration will be done over the "master" surface. The rigid surface is then parameterized by internal coordinates $\alpha^{1}, \alpha^{2}$. Then a point $\mathbf{r}_{s}$ of this surface has to satisfy eqn. (4) as a point in the local coordinate system of the contact element too. This condition leads to the following equation

$$
\mathbf{r}_{s}\left(\alpha^{1}, \alpha^{2}\right)=\boldsymbol{x}\left(\xi^{1}, \xi^{2}\right)+\mathbf{n} \xi^{3}
$$

The 'slave' point projection procedure, which was necessary for the previous description with surface segments, now turns into the determination of the surface point defined by equation (45). Using a "segment-to-segment" type strategy for the computation of the contact integral, first integration points $\xi_{I}^{1}, \xi_{J}^{2}$ are defined on the "master" segment and then the corresponding internal coordinates $\alpha^{1}, \alpha^{2}$ of the rigid surface as well as the penetration $\xi^{3}$ are computed e. g. by the Newton method. For this algorithm we define a function $F\left(\alpha^{1}, \alpha^{2}, \xi^{3}\right)$ with the components given in eqn. (45)

$$
\mathbf{F}=\left[\begin{array}{c}
x_{s 1}-x_{1}-n_{1} \xi^{3} \\
x_{s 2}-x_{2}-n_{2} \xi^{3} \\
x_{s 3}-x_{3}-n_{3} \xi^{3}
\end{array}\right] \text { with } x_{i}=x_{i}\left(\xi^{1}, \xi^{2}\right)
$$

Its derivative with respect to the coordinates $\left(\alpha^{1}, \alpha^{2}, \xi^{3}\right)$ is:

$$
\mathbf{F}^{\prime}=\left[\begin{array}{ccc}
x_{s 1,1} & x_{s 1,2} & -n_{1} \\
x_{s 2,1} & x_{s 2,2} & -n_{2} \\
x_{s 3,1} & x_{s 3,2} & -n_{3}
\end{array}\right]
$$


Then, the Newton iteration procedure reads as follows for iteration step $n$ :

$$
\begin{aligned}
\Delta \boldsymbol{\alpha}_{n} & =\left[\begin{array}{c}
\Delta \alpha_{n}^{1} \\
\Delta \alpha_{n}^{2} \\
\Delta \xi_{n}^{3}
\end{array}\right]=-\left(\mathbf{F}^{\prime}\right)_{n}^{-1} \mathbf{F}_{n}, \\
\boldsymbol{\alpha}_{n+1} & =\boldsymbol{\alpha}_{n}+\Delta \boldsymbol{\alpha}_{n} .
\end{aligned}
$$

\section{Surface of revolution}

If an analytical surface is a surface of revolution described e.g. by NURBS, see [8], it is possible to give a closed form solution for eqn. (48). In the simplest case $f(r)$ can be a plane NURBS curve uniquely projected onto the $r$ axis, see fig. 5. The revolution of the curve about axis $O Z$ gives a surface of revolution. In a Cartesian coordinate system it can be written as

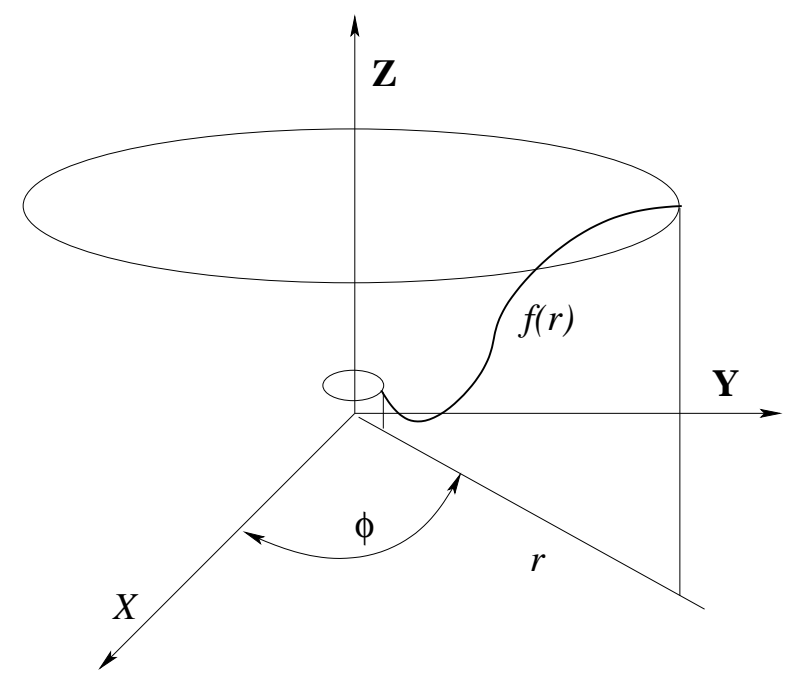

Figure 5: The surface of revolution

$$
\mathbf{r}_{s}(r, \phi)=\left[\begin{array}{c}
x_{s} \\
y_{s} \\
z_{s}
\end{array}\right]=\left[\begin{array}{c}
r \cos \phi \\
r \sin \phi \\
f(r)
\end{array}\right]
$$

Then the iteration vector $\Delta \boldsymbol{\alpha}_{n}$ in eqn. (48) gets the following form:

$$
\Delta \boldsymbol{\alpha}_{n}=\left[\begin{array}{c}
\Delta r_{n} \\
\Delta \phi_{n} \\
\Delta \xi_{n}^{3}
\end{array}\right]
$$


with

$$
\begin{aligned}
& \Delta r_{n}=\frac{1}{D} \cdot\left(\left(x_{3}-f(r)\right)\left(n_{1} \cos \phi+n_{2} \sin \phi\right)+n_{3}\left(r-x_{1} \cos \phi-x_{2} \sin \phi\right)\right) \\
& \begin{array}{r}
\Delta \phi_{n}=\frac{1}{D r} \cdot\left(\left(f(r)-x_{3}-r f^{\prime}(r)\right)\left(n_{1} \sin \phi-n_{2} \cos \phi\right)+f^{\prime}(r)\left(n_{1} x_{2}-n_{2} x_{1}\right)\right. \\
\left.\quad+n_{3}\left(x_{1} \sin \phi-x_{2} \cos \phi\right)\right) \\
\Delta \xi_{n}^{3}=\frac{1}{D r} \cdot\left(f^{\prime}(r)\left(x_{1} \cos \phi+x_{2} \sin \phi-r\right)+f(r)-x_{3}+\xi^{3}\left[f^{\prime}(r)\left(n_{1} \cos \phi+n_{2} \sin \phi\right)-n_{3}\right]\right)
\end{array}
\end{aligned}
$$

and

$$
D=-n_{3}+f^{\prime}(r)\left(n_{1} \cos \phi+n_{2} \sin \phi\right) .
$$

\section{Surfaces allowing a direct computation of the penetration}

For some simple analytical surfaces such as plane surface, cylinder, sphere and torus it is not necessary to solve equation (45) iteratively in order to compute the value of the penetration. In fig. 6 for example a rigid cylinder with the geometry described by the cylinder axis (coordinates $\boldsymbol{x}_{\boldsymbol{m}}$ and direction vector $\boldsymbol{r}$ ) and by the radius $R$ is given. The normal contact gap $g_{N i}$ for the contacting slave point $\boldsymbol{x}_{\boldsymbol{i}}$ can be computed directly subtracting the cylinder radius $R$ from the distance of point $i$ to the cylinder axis.

$$
g_{N i}=|\underbrace{\boldsymbol{x}_{\boldsymbol{m}}-\boldsymbol{x}_{\boldsymbol{i}}+\frac{\boldsymbol{r} \cdot\left(\boldsymbol{x}_{\boldsymbol{i}}-\boldsymbol{x}_{\boldsymbol{m}}\right)}{\boldsymbol{r} \cdot \boldsymbol{r}} \boldsymbol{r}}_{\boldsymbol{v}_{\boldsymbol{n}}}|-R=\sqrt{\boldsymbol{v}_{\boldsymbol{n}}^{\boldsymbol{T}} \boldsymbol{v}_{\boldsymbol{n}}}-R,
$$

where $\boldsymbol{r}$ is a generatrix vector of the cylinder. The derivatives with respect to the variable quantities can be directly computed out of (51):

$$
g_{N i, \boldsymbol{x}_{i}}=-\frac{1}{\sqrt{\boldsymbol{v}_{\boldsymbol{n}}^{\boldsymbol{T}} \boldsymbol{v}_{\boldsymbol{n}}}} \boldsymbol{v}_{\boldsymbol{n}}=\boldsymbol{n}_{\boldsymbol{c}}
$$

and

$$
g_{N i, \boldsymbol{x}_{i} \boldsymbol{x}_{i}}=-\frac{1}{\left(\sqrt{\boldsymbol{v}_{n}^{\boldsymbol{T}} \boldsymbol{v}_{n}}\right)^{3}} \boldsymbol{v}_{n} \boldsymbol{v}_{n}^{\boldsymbol{T}}+\frac{1}{\sqrt{\boldsymbol{v}_{n}^{\boldsymbol{T}} \boldsymbol{v}_{n}}}\left(1-\frac{\boldsymbol{r} \boldsymbol{r}^{\boldsymbol{T}}}{\boldsymbol{r}^{\boldsymbol{T}} \boldsymbol{r}}\right)
$$

The derivatives needed for the tangent matrices with respect to the nodal displacement vector $\boldsymbol{u}_{\boldsymbol{e}}$ are computed using the chain rule with the derivative of geometric approximation $\boldsymbol{x}_{i, \boldsymbol{u}_{e}}$. Within the velocity approach, the corresponding tangent matrix together with the residual is computed at integration points of the contact element, where the value $g_{N i}$ is nonpositive. 


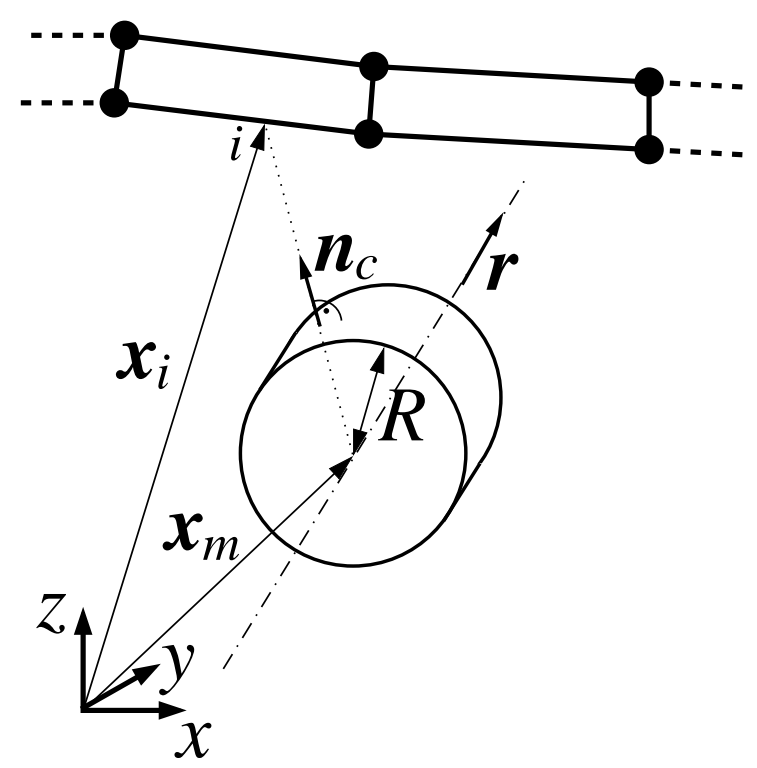

Figure 6: Cylindrical contact surface

\section{Segment-to-analytical surface contact element}

The nodal displacement vector in the case of contact with a rigid surface contains only coordinates of the "master" surface segment

$$
\boldsymbol{u}_{\boldsymbol{e}}^{T}=\left\{u_{1}^{(1)}, u_{2}^{(1)}, u_{3}^{(1)}, u_{1}^{(2)}, u_{2}^{(2)}, u_{3}^{(2)}, \ldots, u_{1}^{(n)}, u_{2}^{(n)}, u_{3}^{(n)}\right\}^{T}
$$

which is taken from the finite element discretization of the deformable body.

Without loss of generality, we can assume the rigid body to be fixed, i.e. with zero velocity $\mathbf{v}_{s}=0$ and variation $\delta \mathbf{r}_{s}=0$. In accordance to that the position matrix $\mathbf{A}$ is modified as

$$
\mathbf{A}=\left[\begin{array}{cccccccccccc}
N_{1} & 0 & 0 & N_{2} & 0 & 0 & \ldots & \ldots & \ldots & N_{(n)} & 0 & 0 \\
0 & N_{1} & 0 & 0 & N_{2} & 0 & \ldots & \ldots & \ldots & 0 & N_{(n)} & 0 \\
0 & 0 & N_{1} & 0 & 0 & N_{2} & \ldots & \ldots & \ldots & 0 & 0 & N_{(n)}
\end{array}\right]
$$

\subsection{Tangent matrix for the penalty approach}

All three cases of contact segments, as described in the previous sections, can be unified by the position matrix A. From equations (14), (15) and (16) we then obtain the following parts of the tangent matrix: 
main part

$$
\begin{aligned}
\mathbf{K}^{(m)} & =\int_{s} \epsilon_{p} H\left(g_{N}\right) \mathbf{A}^{T} \mathbf{n} \otimes \mathbf{n} \mathbf{A} d s= \\
& =\sum_{I, J=1}^{N_{p}}\left(\epsilon_{p} H\left(g_{N}\right) \mathbf{A}^{T} \mathbf{n} \otimes \mathbf{n} \mathbf{A} W_{I} W_{J} \operatorname{det} \mathbf{J}\left(\xi_{I}^{1}, \xi_{J}^{2}\right)\right)
\end{aligned}
$$

rotational part

$$
\begin{aligned}
\mathbf{K}^{(r)}= & -\int_{s} \epsilon_{p} H\left(g_{N}\right) g_{N}\left[\mathbf{A}_{, j}^{T} a^{i j}\left(\mathbf{n} \otimes \boldsymbol{x}_{i}\right) \mathbf{A}+\mathbf{A}^{T} a^{i j}\left(\boldsymbol{x}_{j} \otimes \mathbf{n}\right) \mathbf{A}_{, i}\right] d s= \\
= & -\sum_{I, J=1}^{N_{p}}\left(\epsilon _ { p } H ( g _ { N } ) g _ { N } \left[\mathbf{A}_{, j}^{T} a^{i j}\left(\mathbf{n} \otimes \boldsymbol{x}_{i}\right) \mathbf{A}\right.\right. \\
& \left.\left.+\mathbf{A}^{T} a^{i j}\left(\boldsymbol{x}_{j} \otimes \mathbf{n}\right) \mathbf{A}_{, i}\right] W_{I} W_{J} \operatorname{det} \mathbf{J}\left(\xi_{I}^{1}, \xi_{J}^{2}\right)\right),
\end{aligned}
$$

curvature part

$$
\begin{aligned}
\mathbf{K}^{(c)} & =-\int_{s} \epsilon_{p} H\left(g_{N}\right) g_{N} \mathbf{A}^{T} h^{i j}\left(\boldsymbol{x}_{i} \otimes \boldsymbol{x}_{j}\right) \mathbf{A} d s= \\
& =-\sum_{I, J=1}^{N_{p}}\left(\epsilon_{p} H\left(g_{N}\right) g_{N} \mathbf{A}^{T} h^{i j}\left(\boldsymbol{x}_{i} \otimes \boldsymbol{x}_{j}\right) \mathbf{A} W_{I} W_{J} \operatorname{det} \mathbf{J}\left(\xi_{I}^{1}, \xi_{J}^{2}\right)\right)
\end{aligned}
$$

where $N_{P}$ is a number of integration points; $W_{I}, I=1,2, \ldots, N_{P}$ are weights of the chosen quadrature formula. The determinant of the Jacobian $\operatorname{det} \mathbf{J}\left(\xi_{I}^{1}, \xi_{J}^{2}\right)$ is computed for the surface segment of the "slave" surface in the case of the "segment-to-segment" approach, and for the "master" segment in the case of contact with a rigid surface described by analytical functions.

$$
\operatorname{det} \mathbf{J}\left(\xi_{I}^{1}, \xi_{J}^{2}\right)=\left|\mathbf{r}_{s 1} \times \mathbf{r}_{s 2}\right|_{\xi_{I}^{1}}, \xi_{J}^{2},
$$

where $\mathbf{r}_{s i}, \quad i=1,2$ are corresponding coordinate vectors. In the case of the "nodeto-surface" approach the Jacobian is not necessary, as then no summation over the integration points is needed.

The full normal contact tangent matrix is the sum of all parts

$$
\mathbf{K}=\mathbf{K}^{(m)}+\mathbf{K}^{(r)}+\mathbf{K}^{(c)} .
$$




\section{Residual}

From equation (12) the residual is obtained as

$$
\mathbf{R}=\int_{s} \epsilon_{p}\left\langle g_{N}\right\rangle \mathbf{n}^{T} \mathbf{A} d s=\sum_{I, J=1}^{N_{p}}\left(\epsilon_{p}\left\langle g_{N}\right\rangle \mathbf{n}^{T} \mathbf{A}|\mathbf{J}|_{\xi_{I}^{1}, \xi_{J}^{2}}\right)
$$

\subsection{Element matrices for the Augmented Lagrangian approach}

The Augmented Lagrangian method is also written in a standard form for a surface contact segment with the gap function and the Lagrange parameters evaluated at the integration points; thus the same local elementwise interpolation as for the penalty method can be used. However this leads in general to differences in the contact stresses - stress jumps - between neighboring contact segments. As an alternative the nodal gap values of the surface segments are taken and a corresponding nodal interpolation is assumed for the stress distribution $\lambda$ as e.g. proposed in [1] for the standard Lagrange multiplier method. Then a smooth stress distribution across the segment boundaries is achieved.

Such a 'distributed' contact consideration has definitely some advantages compared to the satisfaction of contact at nodes only, in particular as the nodes of higher order elements have to be treated differently [5]. Nevertheless, in order to achieve a continuous stress distribution and still maintaining stability of the algorithm, certain requirements should be fulfilled as e.g. proposed in [1]. According to the chosen 'Solid-Shell' element type, 4- and 9-node surface segments with bilinear resp. biquadratic interpolation functions are provided in the following. The combined term (eqn. 17) can then be written - for one segment - as:

$$
\Pi_{c}^{e}=\int_{\tilde{a}_{e}} \lambda g_{N}^{e} d \tilde{a}_{e}+\frac{1}{2} \varepsilon_{p} \int_{\tilde{a}_{e}} g_{N}^{e} g_{N}^{e} d \tilde{a}_{e}
$$

In order to account for the change in the size of the contact surface eqn. (62) should be integrated over the current segment surface $a_{e}$. As $a_{e}$ depends on the deformation state, it should also be considered in the linearization process. However, similar to the idea of the augmentation of the Lagrange parameters we propose to use the value $\tilde{a}_{e}$ from the last load step; thus it can be treated as a constant value inside the equilibrium iteration and is updated after convergence for the next load step. The variation of eqn. (62) leads to:

$$
\delta \Pi_{c}^{e}=\int_{\tilde{a}_{e}} \varepsilon_{p} g_{N}^{e} \delta g_{N}^{e}+\lambda \delta g_{N}^{e} d \tilde{a}_{e}
$$




$$
=\delta \boldsymbol{u}_{\boldsymbol{e}} \int_{-1}^{1} \int_{-1}^{1}\left(\varepsilon_{p} g_{N, \boldsymbol{u}_{\boldsymbol{e}}}^{e} g_{N}^{e}+\lambda g_{N, \boldsymbol{u}_{\boldsymbol{e}}}^{e}\right) \operatorname{det} \tilde{\boldsymbol{J}} d \xi d \eta
$$

where $\boldsymbol{u}_{\boldsymbol{e}}$ is the nodal displacement vector according to eqn. (54) and $g_{N, \boldsymbol{u}_{\boldsymbol{e}}}^{e}$ as the partial derivative of $g_{N}^{e}$ with respect to $\boldsymbol{u}_{\boldsymbol{e}}$. The determinant of the Jacobian $\operatorname{det} \tilde{\boldsymbol{J}}$ according to eqn. (59) is computed using the geometry approximation $\tilde{\boldsymbol{x}}$ of the contact element surface. For $\tilde{\boldsymbol{x}}$ the same interpolation is used as for the structural finite element surface discretization using $\tilde{\boldsymbol{x}}_{\boldsymbol{e}}$ as the vector of the discrete nodal coordinates of the updated configuration from the last load step. The linearization of eqn. (63) necessary for the solution of the nonlinear equation system leads to:

$$
\begin{gathered}
\Delta \delta \Pi_{c}^{e}=\frac{\partial \delta \Pi_{c}^{e}}{\partial \boldsymbol{u}_{\boldsymbol{e}}}=\delta \boldsymbol{u}_{\boldsymbol{e}} \int_{-1}^{1} \int_{-1}^{1}\left(\varepsilon_{p} g_{N, \boldsymbol{u}_{e}}^{e} g_{N, \boldsymbol{u}_{e}}^{e}+\varepsilon_{p} g_{N, \boldsymbol{u}_{e} \boldsymbol{u}_{e}}^{e} g_{N}^{e}\right. \\
\left.+\lambda g_{N, \boldsymbol{u}_{e} \boldsymbol{u}_{e}}^{e}\right) \operatorname{det} \tilde{\boldsymbol{J}} d \xi d \eta .
\end{gathered}
$$

Within this linearization the area of contact is assumed to remain constant, thus $\operatorname{det} \tilde{\boldsymbol{J}}$ remains constant. However, the surface is updated in every load step. The tangent stiffness matrix $\boldsymbol{K}_{\boldsymbol{e}}$ and the residuum vector $\boldsymbol{R}_{\boldsymbol{e}}$ of a surface element are determined by integrating eqn. (64) resp. eqn. (63) numerically using e.g. a Gauss integration scheme with $k$ Gauss points:

$$
\begin{gathered}
\boldsymbol{K}_{\boldsymbol{e}}=\sum_{i=1}^{k}\left(\varepsilon_{p} g_{N i, \boldsymbol{u}_{\boldsymbol{e}}} g_{N i, \boldsymbol{u}_{e}}+\varepsilon_{p} g_{N i, \boldsymbol{u}_{\boldsymbol{e}} \boldsymbol{u}_{\boldsymbol{e}}} g_{N i}\right. \\
\left.+\lambda_{i} g_{N i, \boldsymbol{u}_{e} \boldsymbol{u}_{e}}\right) \operatorname{det} \tilde{\boldsymbol{J}}\left(\xi_{i}^{1}, \xi_{i}^{2}\right) W_{i}, \\
\boldsymbol{R}_{\boldsymbol{e}}=-\sum_{i=1}^{k}\left(\varepsilon_{p} g_{N i, \boldsymbol{u}_{e}} g_{N i}+\lambda_{i} g_{N i, \boldsymbol{u}_{\boldsymbol{e}}}\right) \operatorname{det} \tilde{\boldsymbol{J}}\left(\xi_{i}^{1}, \xi_{i}^{2}\right) W_{i},
\end{gathered}
$$

with the updated Lagrange parameters $\lambda_{i}$ :

$$
\lambda_{i(k+1)}^{(n)}=\lambda_{i(k)}^{(n)}+\varepsilon_{p} g_{N i} .
$$

According to so-called Uzawa's algorithm the Lagrange parameters $\lambda_{i}$ are updated for each augmentation step $(k)$ and initialized for every new load step $(n)$ using the values for $\lambda_{i}$ of the last augmentation step of the previous load step $(n-1)$ and $\lambda_{i}=0$ for the first load step (tab. 2). The value $g_{N i}$ denotes the gap value at the Gauss point $i$ with local coordinates $\left(\xi_{i}^{1}, \xi_{i}^{2}\right)$. Depending on the algorithm chosen, the values for $g_{N i}$ are evaluated directly at the Gauss points (see left hand side a) in fig. 7), the so-called local approach, or the interpolation

$$
g_{N i}=\sum_{j=1}^{n} N_{j}\left(\xi_{i}^{1}, \xi_{i}^{2}\right) g_{N j}
$$




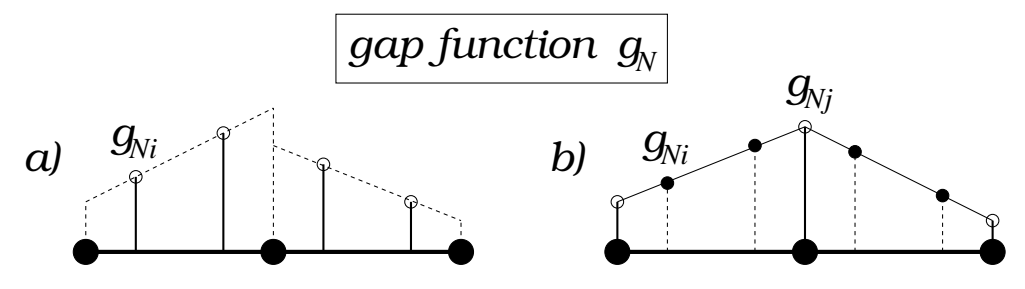

Figure 7: a) Evaluation of gap at Gauss points; b) Evaluation of gap at nodal points and interpolation over segment shape

with the nodal gap values $g_{N j}$ and the bilinear resp. biquadratic shape functions $N_{j}$, the continuous approach, is used (see right hand side b) in fig. 7). According to eqn. (13) $g_{N i}$ is set to zero for values $g_{N i} \geq 0$.

Within the local algorithm the penetration function as well as its derivatives with respect to the nodal displacements are only evaluated at the Gauss points and there is no contribution from Gauss points without penetration. A major advantage of this approach compared to nodal contact is that the number of points, where contact should be considered in an element or segment, can be arbitrarily increased by using a larger number of Gauss points. This allows to follow contact surfaces with strongly varying shape. However, as the gradients of the contact forces are then mostly also larger, a mesh refinement of the contacting body appears to be mandatory, too. For the scheme with the Lagrange parameter being also evaluated resp. augmented at the Gauss points, an interpolation within elements only, fully associated to the penetration interpolation is assumed. Thus the contact stress distribution is not continuous along the contact surface.

Using the continuous approach a smooth contact stress distribution is achieved. Here the penetration function and its derivatives with respect to the nodal displacements as well as the Lagrange parameters are evaluated at the nodal points and interpolated over the surface using the element shape functions according to eqn. (68). However, then the contact refinement as discussed for the local algorithm by using a larger number of Gauss points is not possible. Instead a mesh refinement has to be performed, if an improvement of the contact computation is required, because a better approximation of the geometric characteristics becomes necessary too.

\section{Continuous and local approach for different geometrical situations}

Using a continuous resp. a local approach is similar to the use of different integration methods for the contact terms. Investigations about integration rules for penalty based contact formulations can be found in [18]. In [1] the stability and the performance of different contact algorithms based on Lagrangian multipliers are discussed. To investigate the influence of the integration methods on the fulfillment of the contact constraint some specific geometrical structures are shown where the contact condition 
is formulated using the Augmented Lagrangian scheme. In particular three different geometrical contact situations of a slave structure discretized with bilinear contact segments against different master surfaces are treated.

In the first case shown in tab. 4 contact against a piecewise linear master surface is checked. The penetration can be reasonably limited to the two tested integration

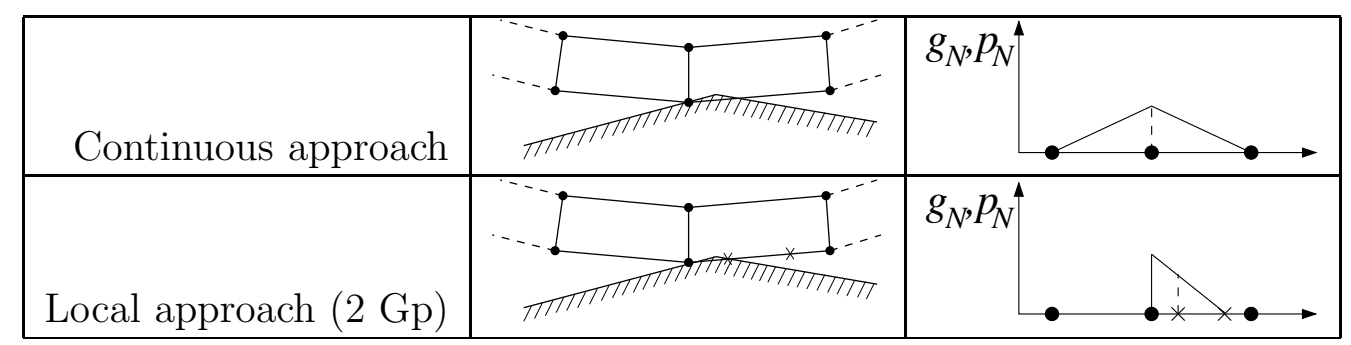

Table 4: Contact against piecewise linear master surface, gap $g_{N}$ and contact pressure $p_{N}$ distribution

schemes. A continuous contact stress distribution over the two involved slave segments is achieved for the continuous approach, which is clearly preferable in this situation. For the local approach with two contact integration points one slave segment is in contact to the master surface with one Gauss point penetrating into the master surface because the Augmented Lagrangian approach prevents a local higher penetration which would result in a distributed contact with some more contact points involved. This leads consequently to a discontinuous stress distribution. Using the local approach with more integration points could prevent a local high penetration. Therefore the local approach should be to preferred.

The second case treats contact against a curved master surface with very small curvature radius compared to the geometry of the slave structure (see tab. 5). In this special geometrical situation large penetration can occur for the continuous approach as well as for the local approach with an overly small number of integration points. In fact the penetration can be limited using the Augmented Lagrangian treatment of contact as it is done here, but the penetration check is only done at single contact integration points or the contacting nodes, so that locally still high penetration values can occur. It's even possible that contact is not found at all. The use of a higher number of contact integration points can help to introduce the contact constraint more properly and to achieve a better contact stress distribution. This can be seen in tab. 5 for the version with 6 contact integration points. With that kind of a contact refinement a very simple way is found to achieve a fairly satisfying introduction of the contact constraint in such special geometrical situations. Alternatively a mesh refinement using more contact segments resp. structural shell elements on the slave side or a subdivision of the segments into subdomains as described in section 3.3 is possible. Changing the master part to the slave part would also lead to a better result in this particular case. 


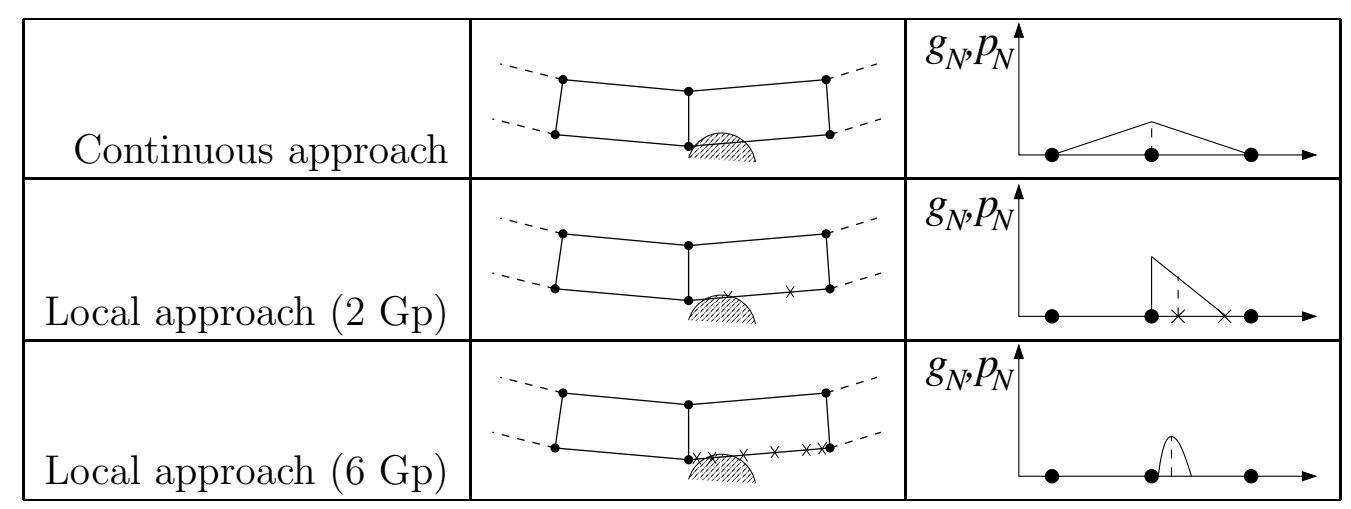

Table 5: Contact against curved master surface, gap $g_{N}$ and contact pressure $p_{N}$ distribution

A third considered case (see tab. 6) is contact on both sides of the shell structure whereas two circular concentric master contact surfaces are assumed. A situation like

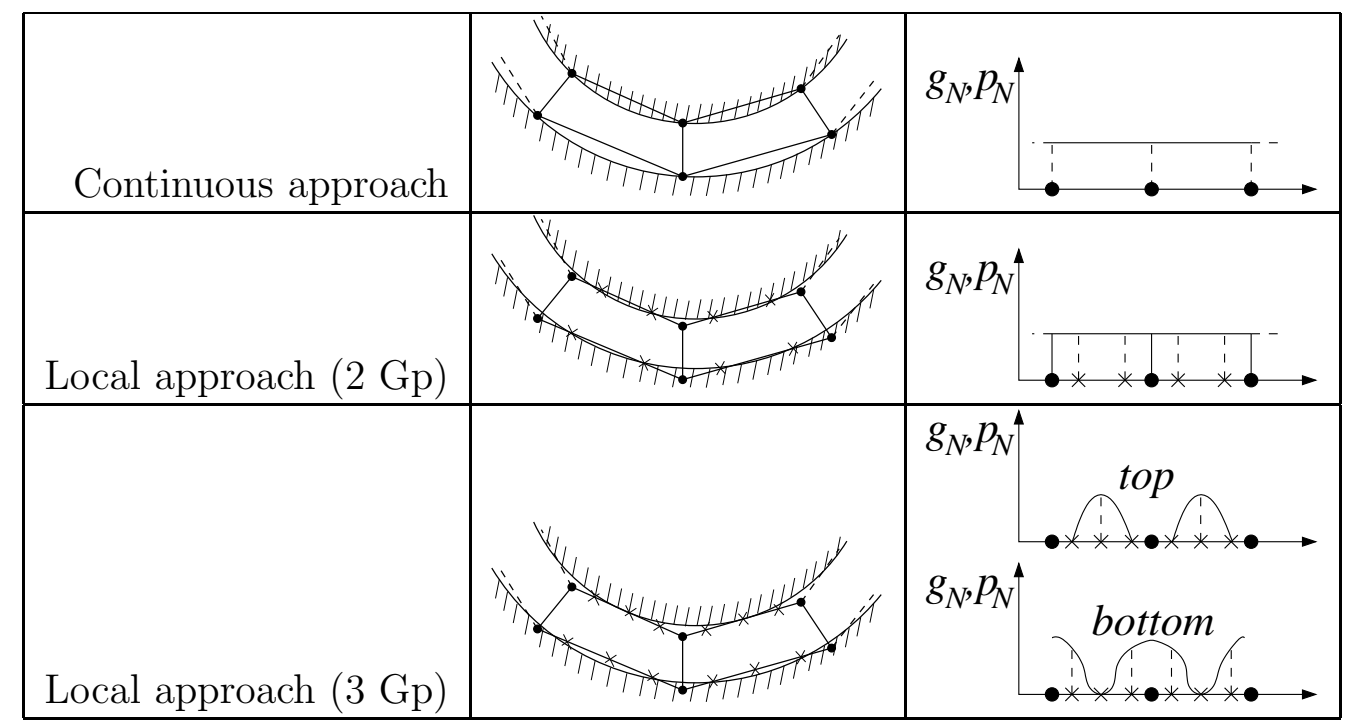

Table 6: Contact between two curved master surfaces, gap $g_{N}$ and contact pressure $p_{N}$ distribution on top resp. bottom of shell in contact

this for example appears in sheet metal forming, where the sheet is formed between two rigid tools. The local approach and the continuous approach with two contact integration points lead to an almost continuous constant stress approach (continuity cannot be guaranteed for the local approach), as the tools are assumed to be described by circles and the blank is meshed with almost equally sized elements. Using the local approach with more than two contact integration points overconstrains the contact problem with an artificial stress distribution, in addition numerical problems will arise in this case. The situation shown in tab. 6 has been also investigated by checking 
the eigenvalues of the stiffness matrix of a single element between two curved contact surfaces. Using two Gauss points for the local approach as well as the continuous approach delivers the same condition number for the stiffness matrix. Increasing the number of integration points for the local approach the condition number increases, too, which indicates the overconstrained situation. Using only one Gauss point leads to an instability for both approaches. Thus it can be seen that it is not reasonable to use the local approach in such a geometrical situation. If the local approach is used the number of integration points has to be chosen compatible to the geometry of the master surfaces.

\section{$5 \quad$ Numerical examples}

\subsection{Patch test}

Here we consider the application of the segment-to-segment contact approach with various integration schemes to the modified patch test problem, originally proposed in Crisfield [6] for the $2 \mathrm{D}$ patch test. The upper block with the dimensions $1 \times 1 \times 0.5$ is meshed with a regular rectangular mesh $3 \times 3 \times 2$. The lower block has the same geometry as the upper block, and a finer, but distorted mesh $6 \times 6 \times 2$ is used, see fig. 8. Both blocks are made of elastic material with the following parameters: Young's modulus $E=1.0 \cdot 10^{5}$, Poisson ratio $\nu=0.3$. The value of the penalty is chosen as $\varepsilon=1.0 \cdot 10^{7}$. During contact the upper block is considered as a slave. An uniform vertical displacement of $\Delta=0.05$ is applied on the top surface.

The patch test serves to check the ability to transfer an uniform stress state through the contact surface. Different techniques were proposed to pass the patch test. Taylor and Papadopoulos [23] proposed the two-pass algorithm based on interchanging the master and slave parts to pass the patch test in the case of a linear approximation for 2D problems. Zavarise and Wriggers [27] proposed the integration over overlapping regions in the $2 \mathrm{D}$ case in order to more accurately treat the contact conditions. Crisfield [6] considered contact elements with higher order approximation to satisfy a patch test in the $2 \mathrm{D}$ case. Also in the $2 \mathrm{D}$ case the integration of the contact integral over the overlapping zone, which is constructed by projection was investigated in El-Abbasi and Bathe [1] to satisfy the patch test. Jones and Papadopoulos [13] considered a special pressure interpolation in the overlapping region of the two contact elements to pass patch test in 3D. Heinstein and Laursen [12] developed an algorithm based on the construction of a special 3D element in the overlapping region for mesh-matching problem as well as to pass the patch test.

The integration algorithm based on integration of subdomains is an approximate approach to integrate discontinuous functions. Here we show, that with this technique it is possible to construct a sequence of results with diminishing error to finally satisfy the 

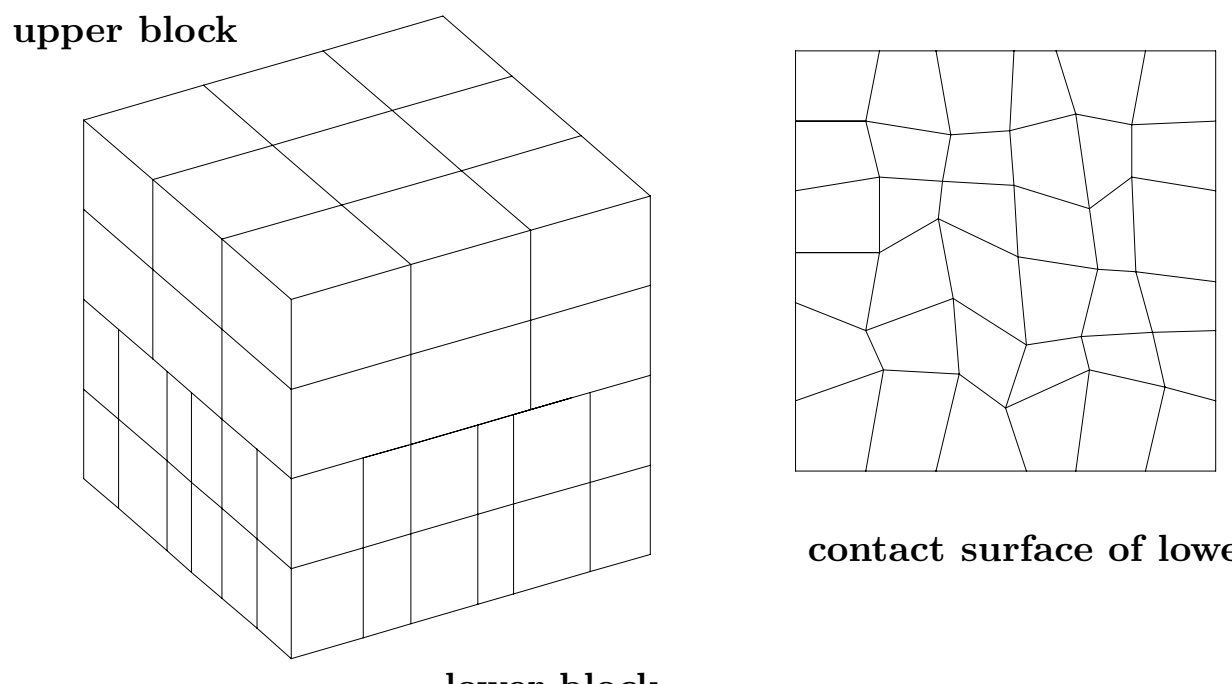

contact surface of lower block

lower block

Figure 8: Blocks for the patch test; Upper block - regular mesh; Lower block - distorted mesh

patch test. In order to investigate in the case of uniform stresses the normal contact traction $N$, the normal stress $\sigma_{z}$ and the vertical nodal displacement $u_{z}$ of the contact surface of the lower block are checked. Their values are controlled by the mean value $\bar{x}$, while the standard deviation $\sigma$ and the coefficient of variation $C_{v}=100 \% \cdot \sigma / \bar{x}$ are computed to estimate the variation. In tab. 7 the results concerning the mean value and the coefficient of variation for the following quantities are given: sum of contact tractions $N=\varepsilon_{p} g_{N}$ over the surface, computed at Gauss points of the contact surface; normal stresses $\sigma_{z}$, computed for the upper and lower surface of each element of the lower block; nodal vertical displacements $u_{z}$ for the contact surface. For comparison, the first computation was made for the node-to-surface approach with a of penalty value of $10^{5}$ which was reduced due to the convergence problems. As is known, this approach fails the patch test. Fig. 9 shows the "roof-like" contact surface of the lower block for this case. As expected this approach leads to the maximum of the coefficient of variation.

From tab. 7 it becomes clear that also for 2D the integration with subdomains leads to - though only slightly - smaller variations than an algorithm with standard Gauss integration. It appears rather remarkable that the variations of the tractions remain constant while the variation of the stresses and displacements falls below one percent. However the effect of the subdivision remains rather small in this case. 


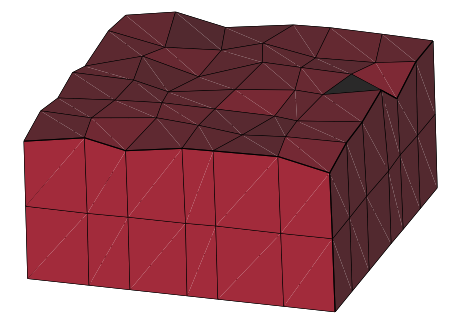

Figure 9: Node-to-surface approach fails to pass the patch test; "roof-like" contact surface

\begin{tabular}{|c|c|c|c|c|c|c|c|}
\hline No. Gpt & No. sbd. & \multicolumn{2}{|c|}{ tractions $N$} & \multicolumn{2}{|c|}{ stress $\sigma_{z}$} & \multicolumn{2}{c|}{ displ. $u_{z}$} \\
\hline & & $\bar{x} \cdot 10^{4}$ & $v \%$ & $\bar{x} \cdot 10^{3}$ & $v \%$ & $\bar{x} \cdot 10^{-2}$ & $v \%$ \\
\hline$N T S^{*}$ & 1 & -1.3084 & -68.087 & -1.5248 & -120.039 & -0.9150 & -157.93 \\
\hline 2 & 1 & -1.7767 & -21.474 & -4.6787 & -2.5772 & -2.4286 & -7.7430 \\
\hline 6 & 1 & -1.6738 & -19.851 & -4.7077 & -1.1663 & -2.4143 & -1.5571 \\
\hline 3 & 2 & -1.6210 & -16.760 & -4.7137 & -0.8570 & -2.4180 & -1.2770 \\
\hline 2 & 3 & -1.6354 & -17.331 & -4.7115 & -0.8789 & -2.4170 & -1.4131 \\
\hline 10 & 1 & -1.6614 & -19.696 & -4.7109 & -0.8595 & -2.4150 & -1.3121 \\
\hline 2 & 5 & -1.6477 & -15.793 & -4.7113 & -0.7798 & -2.4166 & -1.2097 \\
\hline 5 & 2 & -1.6408 & -17.572 & -4.7139 & -0.7942 & -2.4160 & -1.2383 \\
\hline 20 & 1 & -1.6537 & -19.226 & -4.7124 & -0.7791 & -2.4164 & -1.1597 \\
\hline 10 & 2 & -1.6408 & -16.667 & -4.7128 & -0.7585 & -2.4159 & -1.1304 \\
\hline 4 & 5 & -1.6299 & -16.790 & -4.7141 & -0.7136 & -2.4158 & -1.0822 \\
\hline 5 & 4 & -1.6447 & -16.395 & -4.7125 & -0.7239 & -2.4154 & -1.1190 \\
\hline 2 & 10 & -1.6337 & -16.341 & -4.7126 & -0.7578 & -2.4170 & -1.1335 \\
\hline
\end{tabular}

Table 7: Influence of different integration schemes; patch test; mean value and coefficient of variation for the following quantities: contact tractions $N$ on the contact surface, normal stresses $\sigma_{z}$ in the lower block and vertical nodal displacements $u_{z}$ on the contact surface; $N T S^{*}$ - node-to-surface approach

\subsection{Free bending of a metal sheet on two cylinders}

A second example is the free bending problem of a metal sheet (thickness $t$ ) with an elasto-plastic material law with the material data given in fig. 10. At the beginning the metal sheet is positioned on two cylindrical rigid bodies. As loading a displacement $u$ is prescribed in the center of the sheet. Due to symmetry only one half of the system has to be modeled and discretized using 12 bilinear resp. 6 biquadratic elements and a rather fine mesh with 100 bilinear resp. 50 biquadratic elements. Concerning contact the following variations were investigated: 


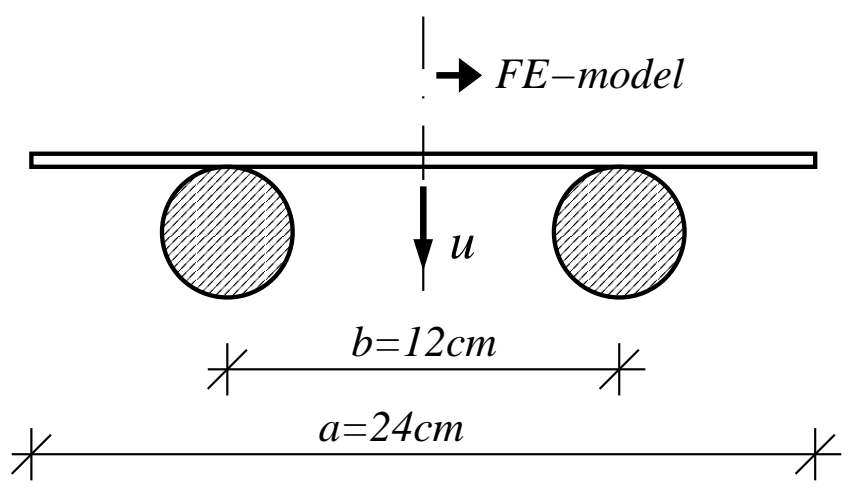

$$
\begin{aligned}
t & =0.25 \mathrm{~cm} \\
\kappa & =1.75 \cdot 10^{4} \frac{\mathrm{kN}}{\mathrm{cm}} \\
\mu & =8.077 \cdot 10^{3} \frac{\mathrm{kN}}{\mathrm{cm}^{2}} \\
y_{0} & =16 \frac{\mathrm{kN}}{\mathrm{cm}} \\
\varepsilon_{p} & =10 \frac{\mathrm{kN}}{\mathrm{cm}^{3}}
\end{aligned}
$$

Figure 10: Geometry and material data of free bending on two cylinders

1) the rigid cylinder is modeled by linear finite elements with 48 elements in the circumferential direction. The metal sheet is treated as "master" part, the cylinder as "slave" part. Contact is modeled by the "node-to-surface" approach.

2) the model is the same as in 1 , but contact is modeled by the "segment-to-segment" approach.

3) the surface of the rigid cylinder is described analytically, the metal sheet is then the "master" part. The contact integral is computed by the nodal collocation formula with additional gap interpolation over the element surface.

4) the model is the same as in 3. The Gauss quadrature formula with different numbers of integration points on the "master" part is used.

In fig. 11 the results for the global central reaction force for case 1 , case 2 with $2 \times 2$ Gauss points and case 3 each with a mesh of 12 bilinear elements are plotted. The result with the fine mesh of 100 bilinear ANS3DEAS elements with one integration point for contact evaluation is taken for comparison as "exact" solution. The nodal collocation formula of case 3 shows the largest oscillations, because the value of the penetration is checked only at the nodes of the sheet and the mesh is relatively coarse in comparison with the geometrical size of the cylinder despite the analytical description of the cylinder. If contact is checked at nodes of the mesh of the rigid cylinder, in case 1 of the "nodal-to-surface" approach, jumps appear with smaller amplitudes. This is an obvious consequence of the finer mesh for the cylinder in comparison with the sheet mesh. As is well known, this would improve with a finer mesh on both sides. Using a Gauss quadrature for the "segment-to-segment" strategy leads to only slightly reduced jumps, because in both "node-to-surface" and "segment-to-segment" approach the rigid cylinder is modeled still with a rather coarse finite element mesh. In order to investigate the influence of the order of the Gauss integration, case 4 was extended with $2 \times 2,3 \times 3,7 \times 7$ and $10 \times 10$ integration points and, finally, compared to the results with a refined mesh of 100 elements, but with 1 Gauss point only, see fig. 12. Obviously, the quadrature formulae with $2 \times 2$ integration points leads to rather large oscillations. 


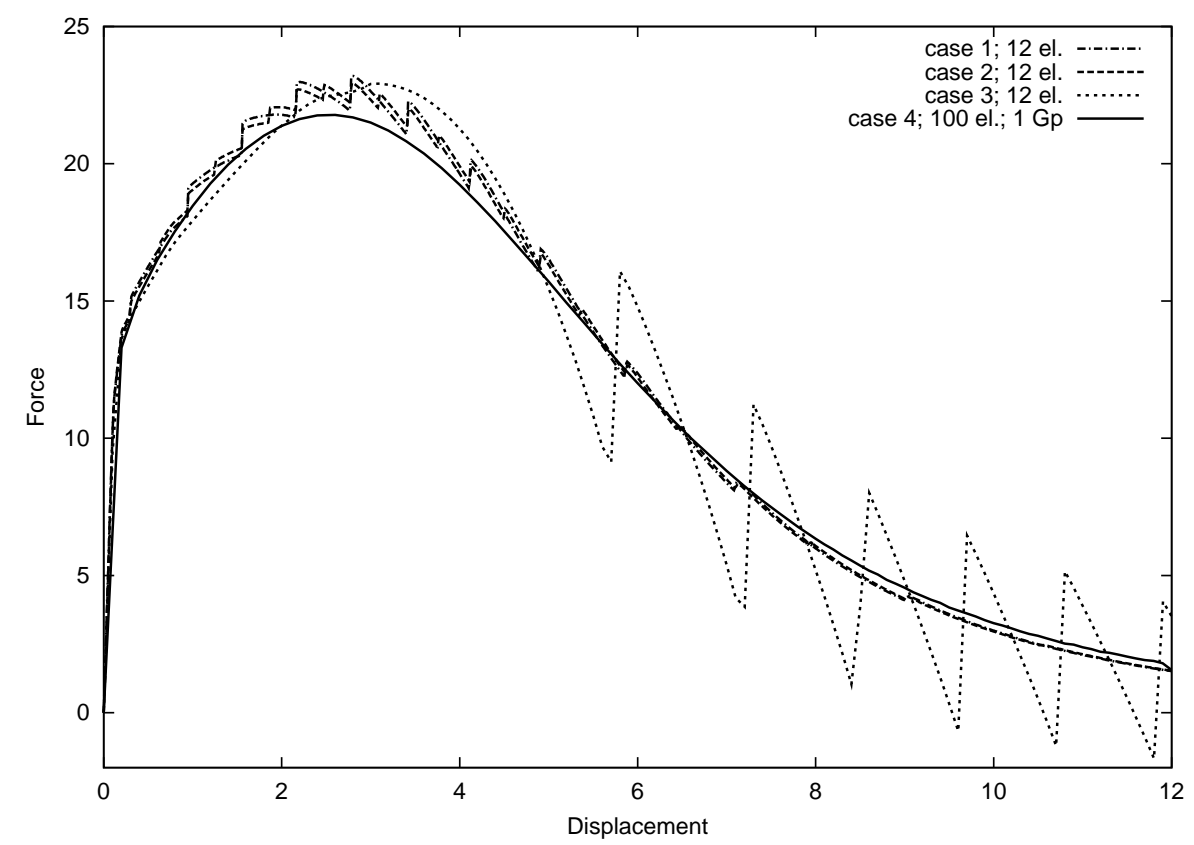

Figure 11: Force-deflection curves for free bending problem; bilinear ANS3DEAS elements; comparing quadrature formulae of low order and mesh refinement

This is due to the fact that rather non-smooth contact checking is performed, which can be improved using more contact points. As mentioned earlier, checking contact at Gauss points can be interpreted as integration of a discontinuous function for which no a-priori error estimation is avaliable. One can see that a convergent sequence of curves is achieved if the number of integration points is increased even in the case of a coarse mesh for the sheet. The influence of the mesh refinement with a softer response of the sheet is also obvious.

The next step is to consider the influence of the number of Gauss points for the sheet meshed with biquadratic elements. Fig. 13 shows the result of the computation for the beam meshed with 6 elements, if the integration formula with $6 \times 6$ integration points is used and if as an alternative the integration formula with 2 subdomains and $3 \times$ 3 integration points in each and with 3 subdomains and $2 \times 2$ integration points are used. The result is compared with a refined mesh of 12 elements for the beam meshed with 12 elements with $3 \times 3$ integration points. For comparison a 50 element mesh and $2 \times 2$ integration points per element is chosen. The density of the Gauss points to check the penetration is identical in the first three cases, but as a consequence of the smallest a-priori integration error for the algorithm with subdomains, the latter leads to a reduction of the oscillations. In fig. 14 it is shown that even a relatively large number of $10 \times 10$ Gauss points per element still preserves oscillations. The integration with 5 subdomains and 2 Gauss points for the 6 element mesh leads again to a solution with a smaller deviation. 


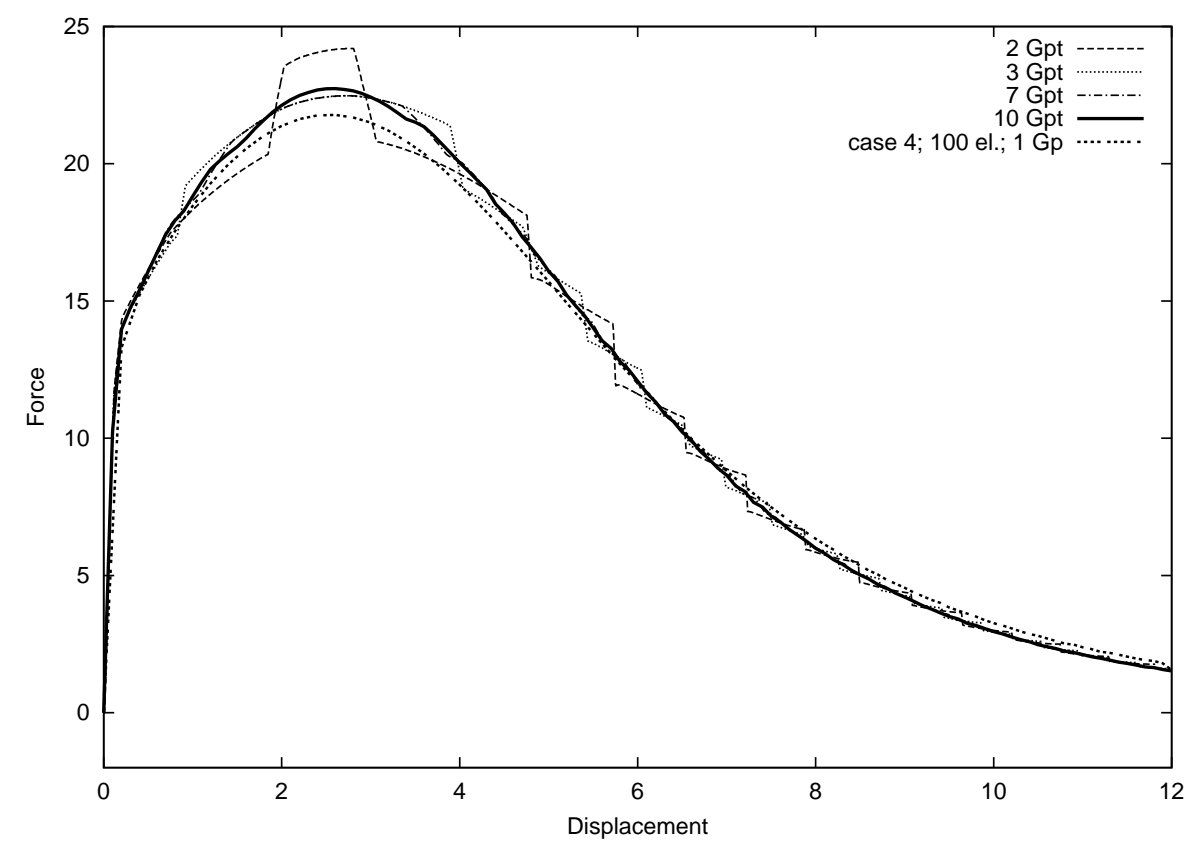

Figure 12: Force-deflection curves for free bending problem; bilinear ANS3DEAS elements; contact against analytically defined contact surface; influence of the number of Gauss points

\subsection{Bending between two contacting surfaces}

The third example is a bending problem of a metal sheet (thickness $t$ ) between two contacting bodies both described by two plane surfaces each (see fig. 15). This problem is chosen to show the necessity of the 'Solid-Shell' approach as well as the necessity of the Augmented Lagrangian method. Again the symmetry properties of the structure can be used and only half of the structure has to be discretized with finite elements. Altogether 40 bilinear (ANS3DEAS) resp. 20 biquadratic elements (MI9K3DEAS) are used for the discretization, with a local refinement in the middle of the sheet (fig. 16) where a high gradient in the deformation is expected. An elasto-plastic material law has been used with the material data given in fig. 15 .

This example is also chosen to compare the effort of the penalty method with the Augmented Lagrangian method. For the penalty method penalty parameters of $\varepsilon_{p}=$ $50 \frac{\mathrm{kN}}{\mathrm{cm}}$ and $\varepsilon_{p}=100 \frac{\mathrm{kN}}{\mathrm{cm}}$ have been selected, such that only a small penetration occurs. Using a smaller value for $\varepsilon_{p}$ the penetration becomes unacceptably large, for a higher value numerical problems appear. For the Augmented Lagrangian approach the starting value for the penalty parameter is chosen to $\varepsilon_{p}=10 \frac{\mathrm{kN}}{\mathrm{cm}^{3}}$. This fairly large value is taken for $\varepsilon_{p}$ because otherwise too many augmentation steps would be needed to achieve the maximum penetration limit. For the latter a maximum penetration tolerance of pen $_{\max }=0.05 \mathrm{~mm}$ is assumed. To check the contact condition two contact 


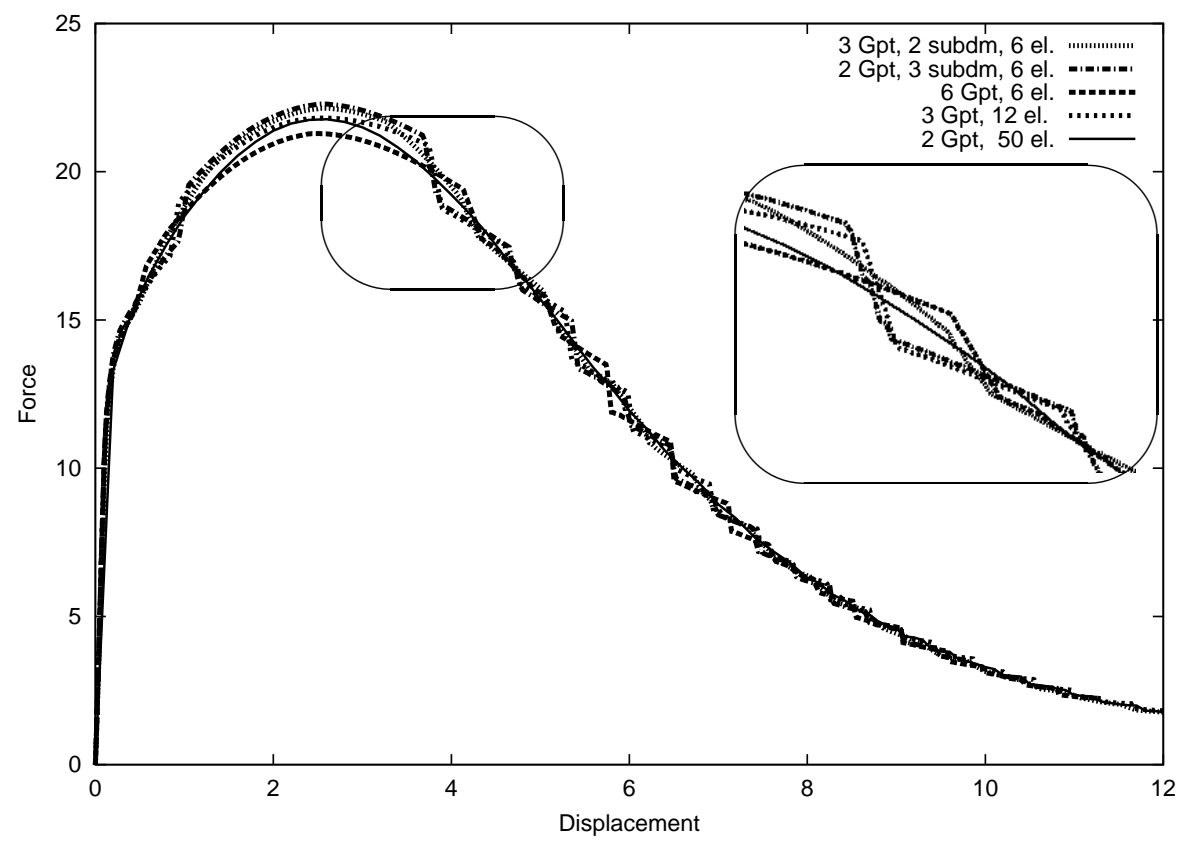

Figure 13: Force-deflection curves for free bending problem; biquadratic MI9K3DEAS elements; Influence of the number of Gauss points and refined mesh

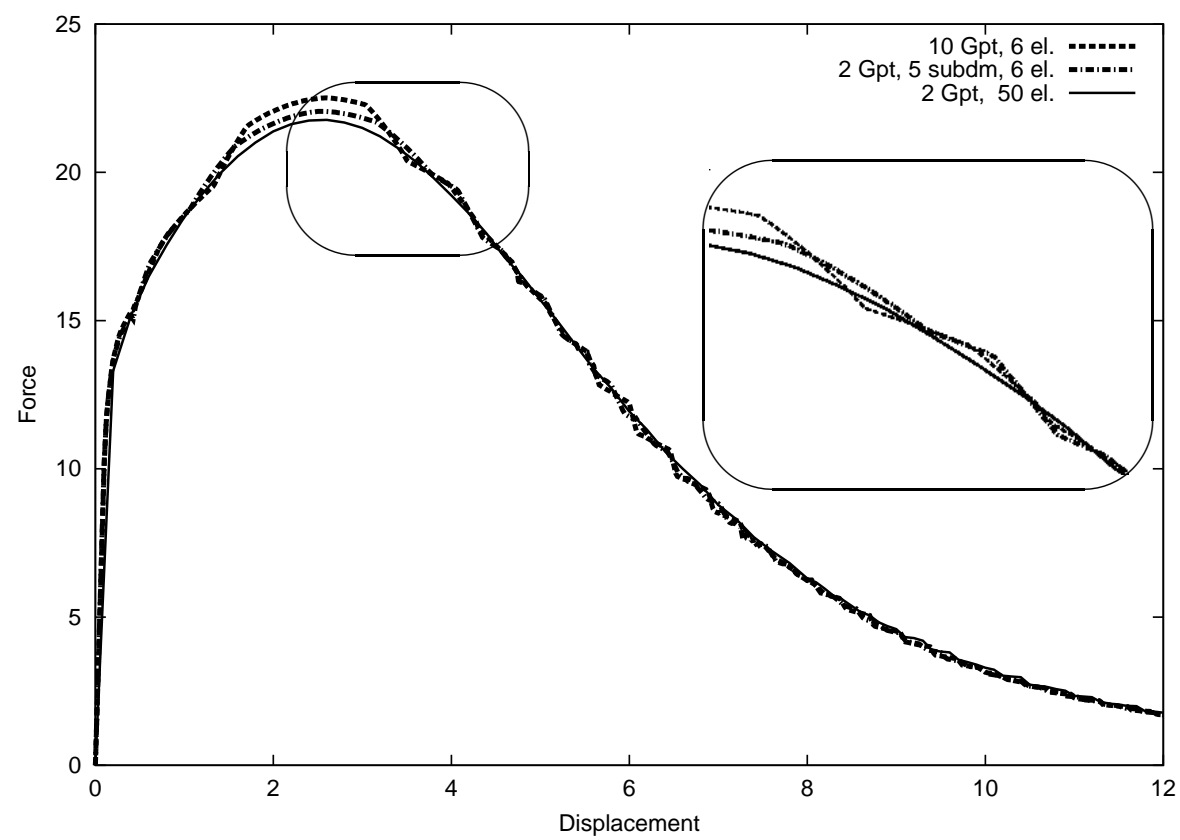

Figure 14: Force-deflection curves for free bending problem; biquadratic MI9K3DEAS elements; subdivision of the integration domain into subdomains results in smaller oscillations 


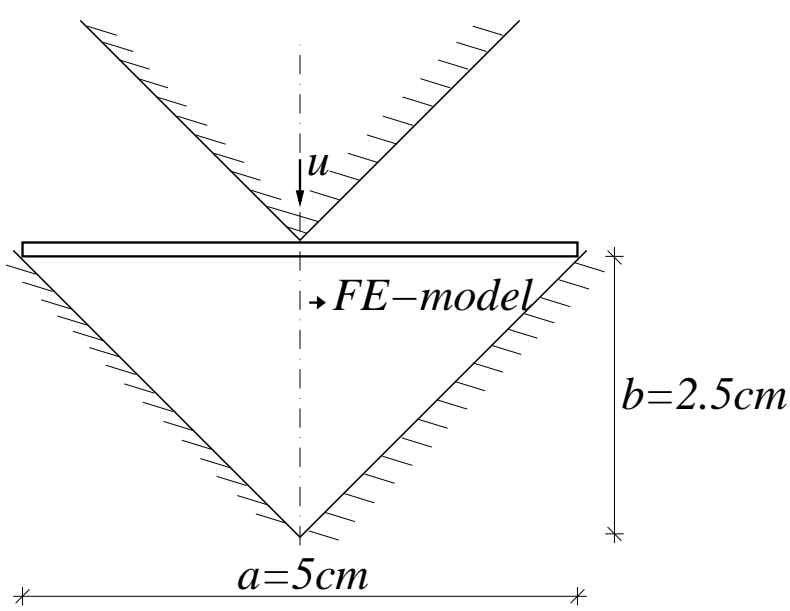

$$
\begin{aligned}
t & =0.1 \mathrm{~cm} \\
\kappa & =1.75 \cdot 10^{4} \frac{\mathrm{kN}}{\mathrm{cm}^{2}} \\
\mu & =8077 \frac{\mathrm{kN}}{\mathrm{cm}} \\
y_{0} & =16 \frac{\mathrm{kN}}{\mathrm{cm}^{2}}
\end{aligned}
$$

Figure 15: Geometry and material data of free bending problem

integration points for the bilinear elements, resp. three for the biquadratic elements, are used in each surface contact segment. An improvement of the results using more Gauss points can not be achieved in this case because of the plane contact geometry.

In the beginning of the deformation process the contact force is very small as the sheet can slide into the structure with little resistance. Only beyond a displacement of $u=2.05 \mathrm{~cm}$ the forces grow considerably. Up to a displacement of about $u=2.2 \mathrm{~cm}$ both approaches lead to almost the same result, as it can also be seen in the forcedeflection curve in fig. 17, where the resulting force in the middle of the sheet is given over the displacement $u$. For displacements of $u>2.2 \mathrm{~cm}$ the penalty approach allows a fairly high penetration, therefore the resulting contact forces are much smaller in this range than those for the Augmented Lagrangian approach. In fig. 16 the deformation state with the maximum penetration for both algorithms is depicted. Using the Augmented Lagrangian approach any penetration can be avoided almost completely. It must also be mentioned that only linear convergence is achieved for the Augmented Lagrangian parameters inside the Augmented Lagrangian iteration algorithm. Therefore many additional iteration steps are required. Up to a displacement of $u=2 \mathrm{~cm}$ the total number of iteration steps is about 450 for the penalty approach; for the Augmented Lagrangian approach about 540 iterations are needed. For a displacement up to $u=2.4 \mathrm{~cm}$ with the penalty approach a total number of iterations of about 2440 is necessary; the Augmented Lagrangian approach requires then about 3870 iterations. Thus it can be concluded that the accurate satisfaction of the constraint condition can be only achieved with high computational effort. For a displacement $u>2.35 \mathrm{~cm}$ also a difference between the biquadratic and the bilinear elements becomes visible. In this range of deformation the biquadratic shape functions seem to be more suitable for approximating the strongly deformed geometry. 


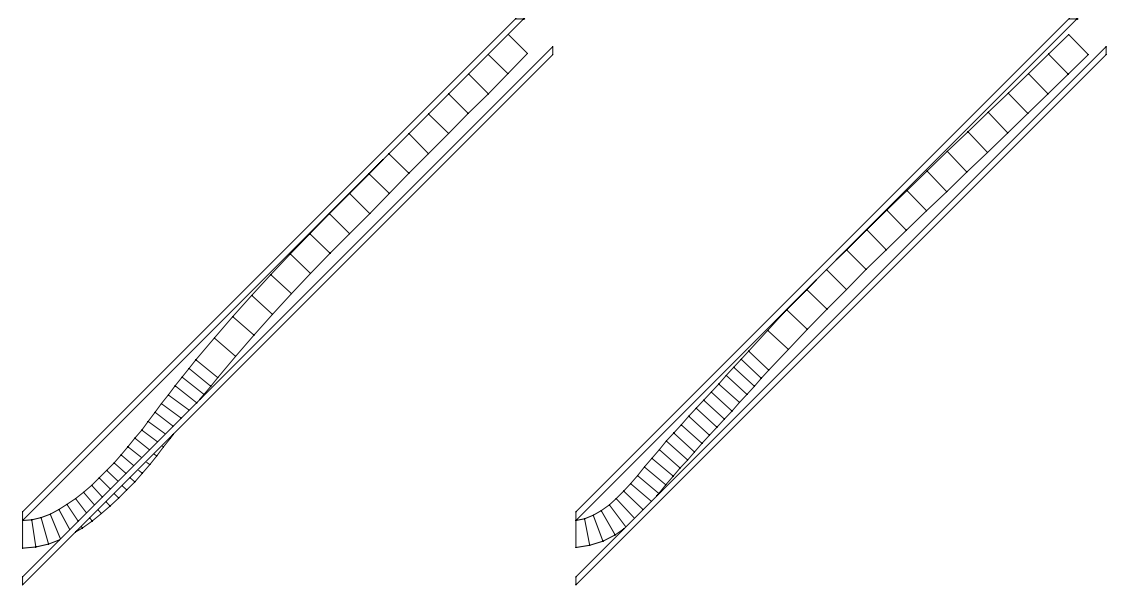

Figure 16: Comparing the state of deformation at $u=2.4 \mathrm{~cm}$ for penalty method (left) and Augmented Lagrangian approach (right)

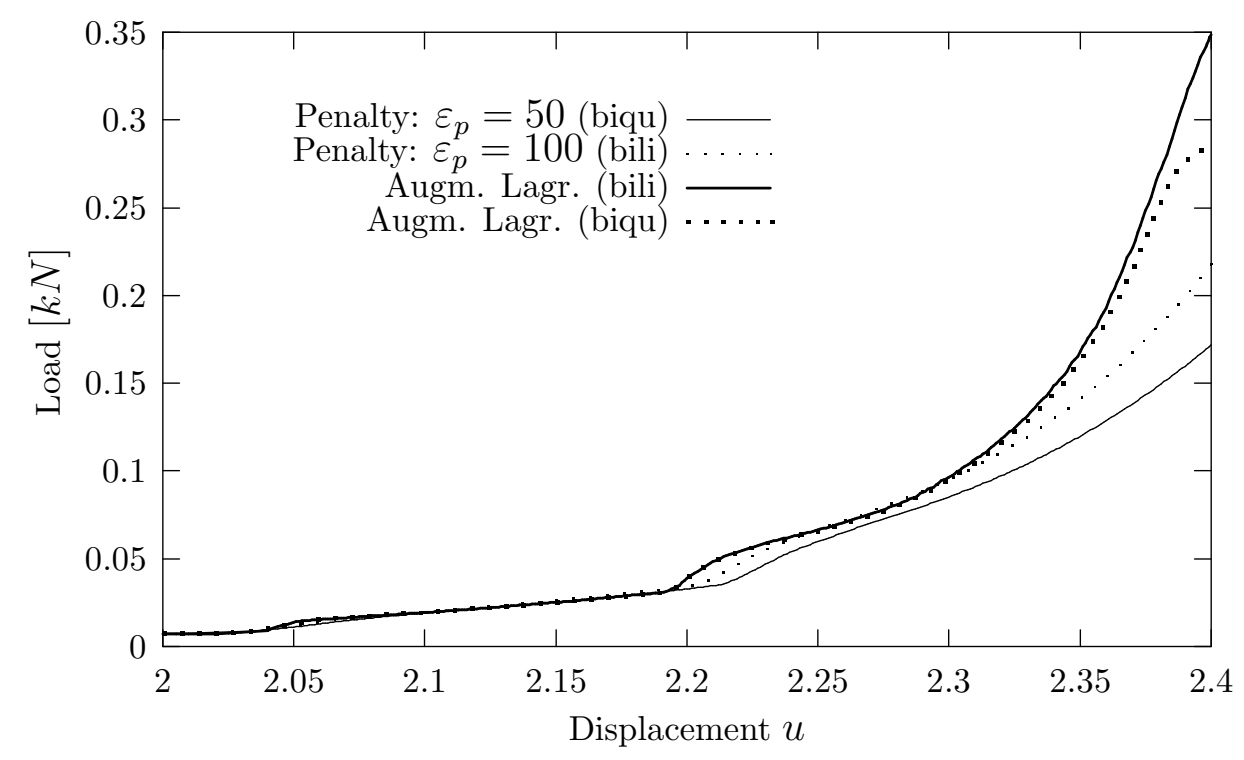

Figure 17: Load-deflection curve for $2 \mathrm{~cm} \leq u \leq 2.4 \mathrm{~cm}$

In fig. 18 the contact stresses for the displacement state with $u=2.38 \mathrm{~cm}$ in the area of $0.1 \mathrm{~cm}<x<0.8 \mathrm{~cm}$ are shown. As expected for the penalty case with higher penetration there are smaller stresses in a wider area, whereas for the Augmented Lagrangian case higher stresses are concentrated on a much smaller region. 


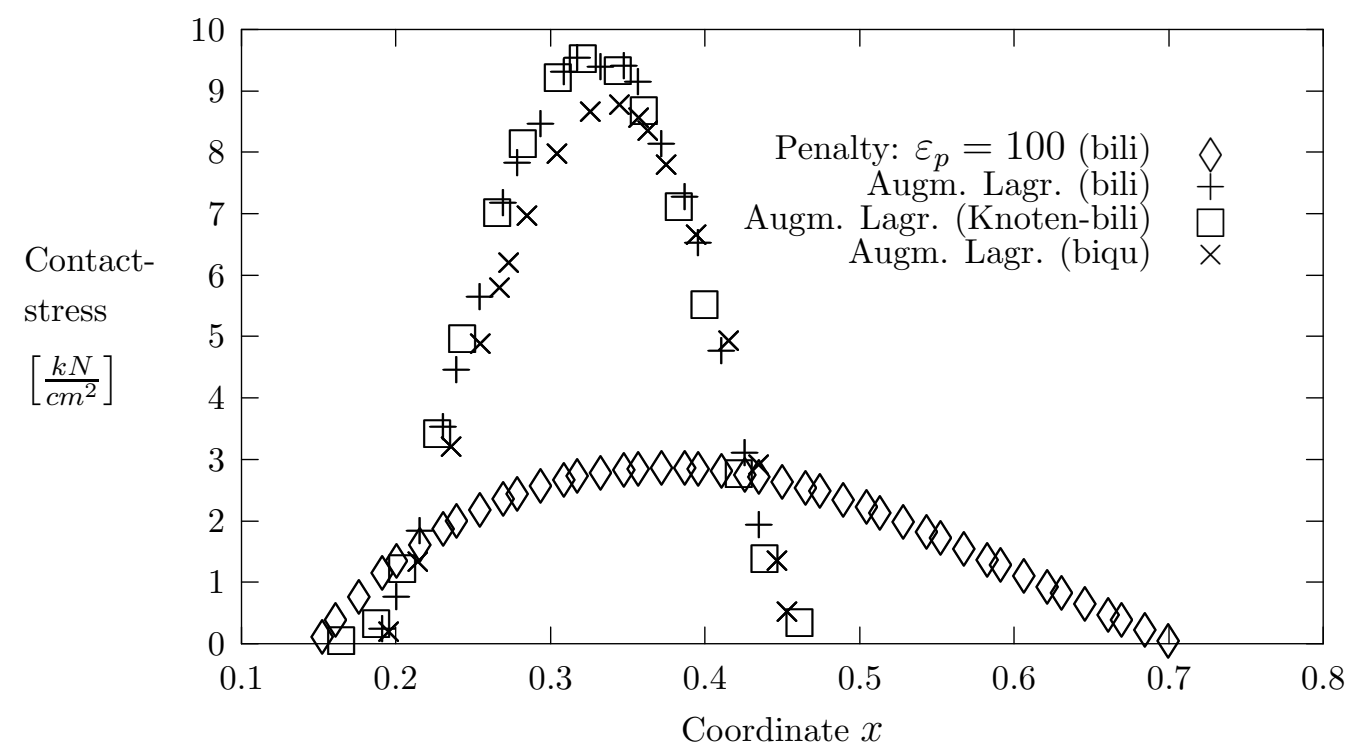

Figure 18: Contact stresses on lower side of the sheet for $u=2.38 \mathrm{~cm}$

\subsection{Deep drawing of a circular cylindrical part with a counter die}

In this numerical example the deep drawing process of a cylindrical part with counter die is simulated. The idea is to show contact for both sides of the sheet which results in a stretching process, if friction is involved, the latter however being excluded in this contribution. The cylindrical sheet has a uniform thickness $t=1 \mathrm{~mm}$ and a radius $R_{S}=8 \mathrm{~cm}$. The geometry of the tools is shown in fig. 19. Due to symmetry only a quarter of the structure is discretized using 469 nodal points (see fig. 20). To describe the contact surfaces of the rigid tools analytically defined surfaces as plane surface, cylinder and torus are combined. Accounting for the geometrical situation the so-called continuous approach is used for the contact description according to section 4.5. Therefore the contact condition is evaluated at the nodal points and the values at the contact integration points are computed by nodal interpolation. Alternatively also a test is performed using the local approach with two contact integration points. Furthermore the Augmented Lagrangian approach with a penalty value starting with $\varepsilon_{p}=20 \frac{\mathrm{kN}}{\mathrm{cm}^{3}}$ and a maximum penetration of pen $n_{\max }=0.01 \mathrm{~cm}$ is used.

As loading a displacement $u$ is prescribed for the punch. The complete forming process can be separated in two phases. In phase 1 the punch presses the sheet against the rounded edges of the die until a displacement of $u=2.5 \mathrm{~cm}$ is reached where the lower surface of the sheet is pressed against a fixed blankholder, the counter die. In phase 2 the punch and the counter die move simultaneously and the blank is drawn along the outer cylindrical die. 


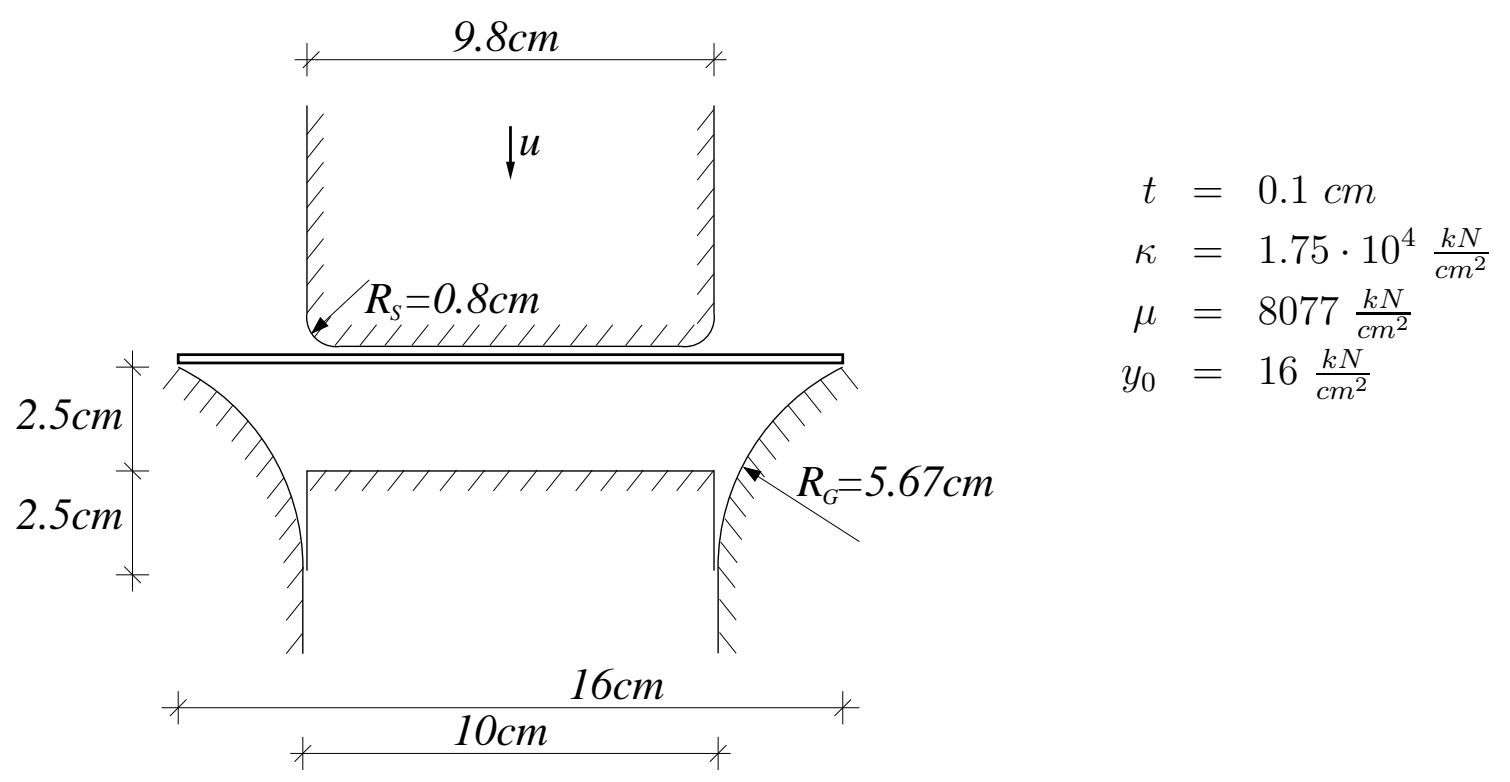

Figure 19: Geometry- and material data for deep drawing process

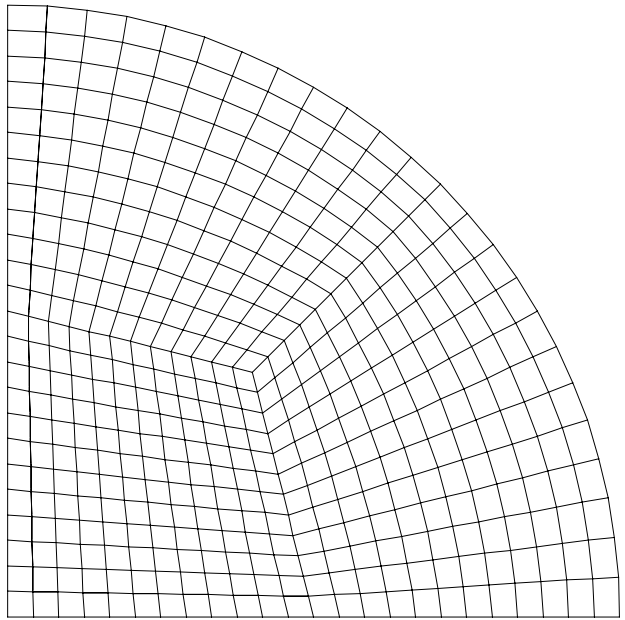

Figure 20: Undeformed mesh for a quarter of the circular sheet

In fig. 21 the contact stresses for the surfaces of the punch, the die and the blankholder are shown. In the first state for $u=2.15 \mathrm{~cm}$ the work piece is pressed by the punch against the die; there is still no contact to the blankholder. From $u=2.5 \mathrm{~cm}$ on the blank is pressed against the blankholder. Thus for the other states also contact stresses for the blankholder appear. In the last state it can be seen that the blank is predominantly drawn along the upper part of the die and the punch. It is also rather obvious that for the bilinear interpolation of the surface geometry for the work piece 
a)
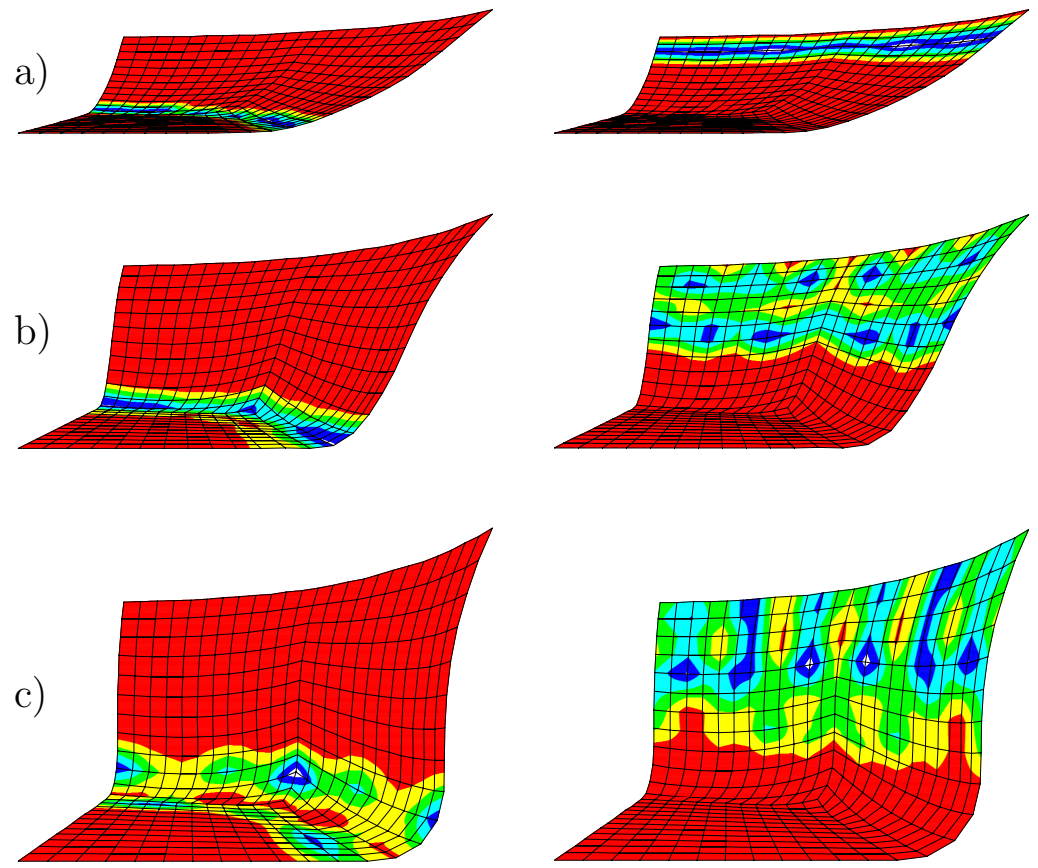

c)
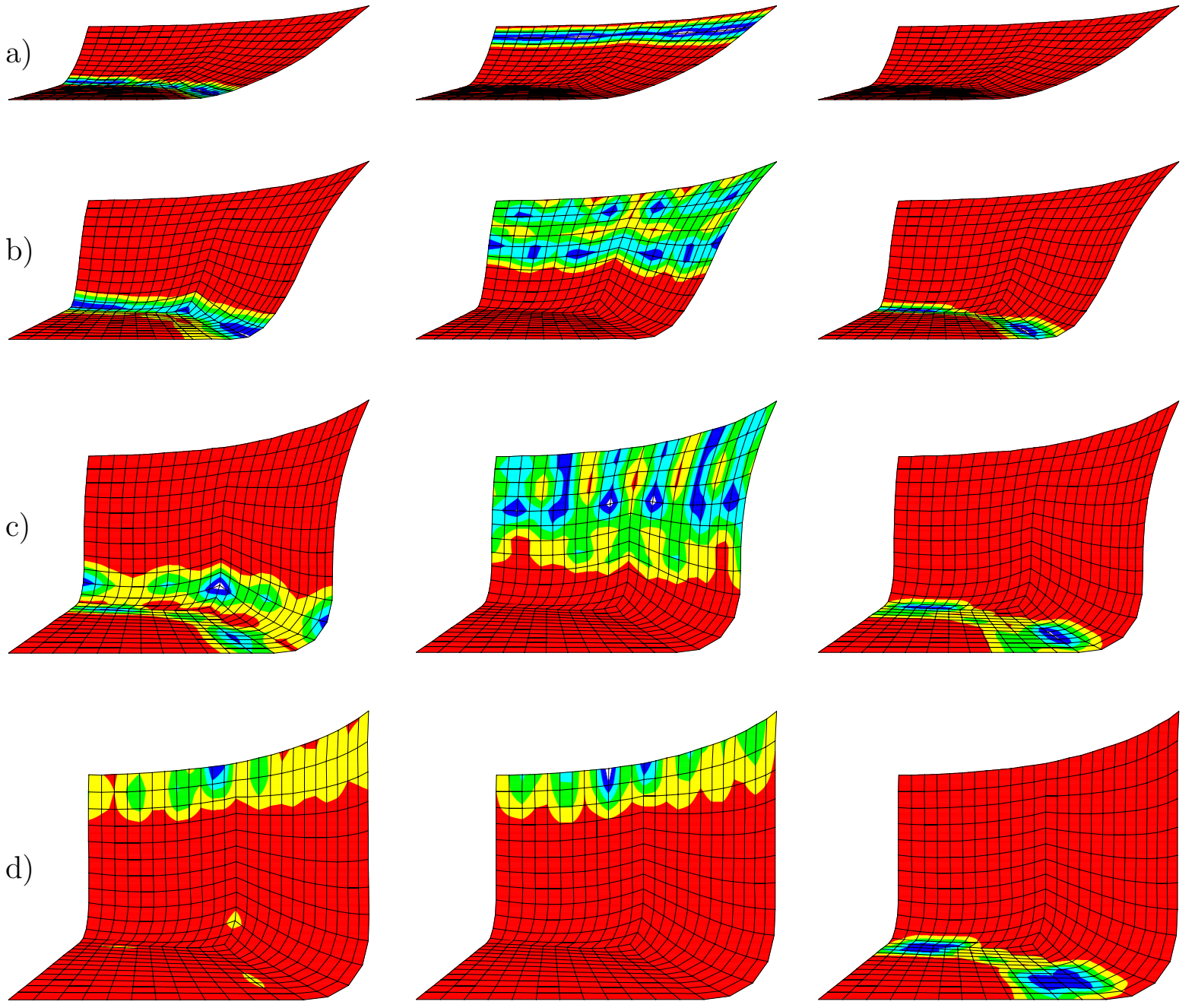

Figure 21: Contact stresses for punch (left), die (middle) and blankholder for a) $u=$ $2.15 \mathrm{~cm}, \mathrm{~b}) u=4.3 \mathrm{~cm}, \mathrm{c}) u=6.45 \mathrm{~cm}$ and d) $u=8.6 \mathrm{~cm}$

the contact stresses are fairly nonuniform for this rather coarse mesh.

In fig. 22 the load-displacement curve for the punch form is shown. The change from phase 1 to phase 2 is marked by a vertical dotted line at $u=2.5 \mathrm{~cm}$. It is obvious that after contact with the counter die the punch force increases until the maximum is reached, when the major bending is achieved and only some stretching is performed due to contact at the upper part of the punch and the outer die. Using the local approach with two contact Gauss points for this test leads to similar but clearly stiffer results. Using the selective reduced integration of volumetric parts (ANS3DEAS-rv) leads to little softer result which indicates that incompressibility locking in the plastic 


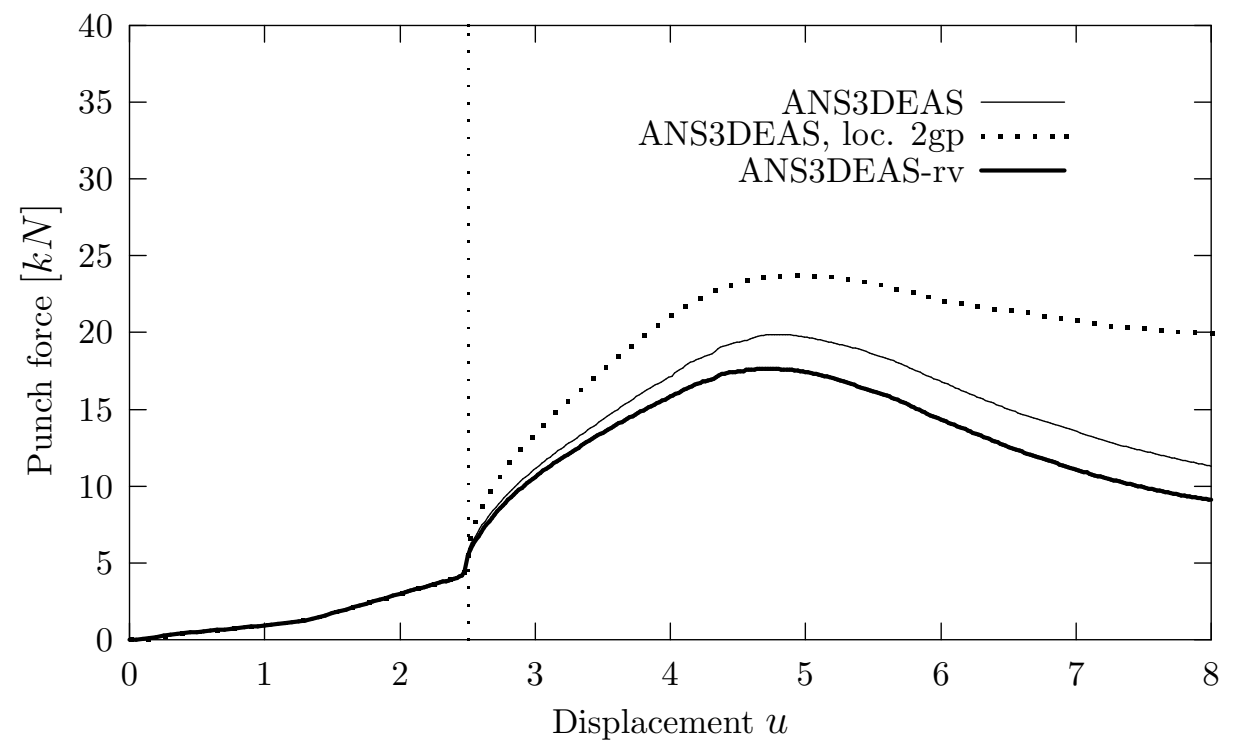

Figure 22: Load-displacement curve for deep drawing process of cylindrical pot

range has to be taken into account. As additional investigation a further refinement of the mesh would be helpful to get information about the convergence properties. 


\section{Conclusions}

In the present paper structural finite 'Solid-Shell' elements have been used for applications in large deformation contact analysis. It was shown that the biquadratic elements are slightly superior concerning the results and using the same number of degrees of freedom. In addition it is advantageous to use thick shell elements with a continuum like geometric interpolation. This allows to use the upper and lower surfaces directly in contact analysis and in addition thickness effects are more properly represented than with usual shell elements.

The main part of the paper is focusing on the comparison of the contact algorithms used especially for problems in sheet metal forming. Several contact approaches are generalized under the unified description based on the special treatment of the contact conditions. Within this treatment different approaches for computation of the contact integral lead to the various contact "elements": the node-to-surface approach with evaluating data either at the nodal points only or with additional interpolation over the contact segment; the segment-to-segment approach allows a choice of the integration rule; the use of rigid contact surfaces described by analytical functions allowing also the choice of the integration rule. In all cases within the choice stability problems must be avoided. The contact approach with analytical functions appears especially useful for applications in sheet metal forming where rigid tools are commonly defined by arbitrary smooth geometries, e.g. by NURBS etc. In this case the well-known closest point procedure can be turned into the computation of the value of penetration from the surface equations directly.

The discussed contact elements with different degree of geometrical approximation of the contact surfaces were tested in the example of a free bending of metal sheet with large sliding in contact. A force-deflection curve was chosen to represent the main characteristics of the results. Large oscillations appeared if relatively coarse meshes together with a low order integration are used. It was found that increasing the number of integration points leads to a more improved reduction of these oscillations, but an integration procedure with an additional subdivision into subdomains leads to a further reduction of oscillations with the same total number of Gauss points over the contact area. However, it becomes also obvious that sufficiently fine meshes are mandatory to achieve good results. It was shown that within the segment-to-segment approach together with an integration over subdomains it is possible to efficiently improve the patch test results.

As regularization techniques for the enforcement of contact conditions the penalty method as well as the Augmented Lagrangian method are used. With some numerical examples the advantages of both methods were compared. For the penalty method small penetrations have to be tolerated with the advantage of efficiency compared to the Augmented Lagrangian approach where the maximum penetration can be almost arbitrarily limited. The latter is particularly necessary in cases, where a strong con- 
straining appears, e.g. in metal forming when sliding about sharp corners occurs or stretching has to be simulated. It appears to be an efficient technique to work with the penalty method checking for an allowable penetration and switch to an Augmented Lagrangian procedure only if such a tolerance is passed.

Finally an example modeling the deep drawing process of a cylindrical part is shown. Here the ability of the 'Solid-Shells' to simulated complex forming processes together with the introduced contact formulations, here particularly the Augmented Lagrangian treatment with so-called continuous approach, can be shown. Two different mesh densities have been tested and different 'Solid-Shells' element variants have been compared for the coarser mesh.

\section{References}

[1] N. El-Abbasi and K.J. Bathe. Stability and patch test performance of contact discretization and new solution algorithm. Comp. Struct., 79(16):1473-1486, 2001.

[2] K.J. Bathe and E. Dvorkin. A continuum mechanics based four-node shell element for general nonlinear analysis. Engng. Comput., 1:77-88, 1984.

[3] P. Betsch and E. Stein. An assumed strain approach avoiding artificial thickness straining for a non-linear 4-node shell element. Commun. Numer. Methods Engng., 11:899-909, 1995.

[4] E.N. Bucalem and K.J. Bathe. Higher-order MITC general shell elements. Int. J. Numer. Methods Engng., 36:3729-3754, 1993.

[5] R. Buczkowski, M. Kleiber, and U. Gabbert. On linear and higher order standard finite elements for 3d-nonlinear contact problems. Comp. Struct., 53(4):817-823, 1994.

[6] M.A. Crisfield. Re-visiting the contact patch test. Int. J. Numer. Methods Engng., 48:435-449, 2000.

[7] PJ. Davis, and P. Rabinowitz. Methods of numerical integration. 2'nd edition; Third Edition, Academic Press, Inc., 1984.

[8] G.E. Farin. NURBS : from projective geometry to practical use. 2'nd edition, Natick, Massachusetts: Peters, 1999.

[9] R. Hauptmann, S. Doll, M. Harnau, and K. Schweizerhof. 'Solid-Shell' elements with linear and quadratic shape functions at large deformations with nearly incompressible materials. Comp. Struct., 79(18):1671-1685, 2001. 
[10] R. Hauptmann and K. Schweizerhof. A systematic development of solid-shell element formulations for linear and nonlinear analyses employing only displacement degrees of freedom. Int. J. Numer. Methods Engng., 42:49-70, 1998.

[11] R. Hauptmann, K. Schweizerhof, and S. Doll. Extension of the solid-shell concept for large elastic and large elastoplastic deformations. Int. J. Numer. Methods Engng., 49:1121-1141, 2000.

[12] M.W. Heinstein, T.A. Laursen. A three dimensional surface-to-surface projection algorithm for non-coincident domains. Int. J. Numer. Methods Engng., 19:421432, 2003.

[13] R.E. Jones and P. Papadopoulos. A novel three-dimensional contact finite element based on smooth pressure interpolations. Int. J. Numer. Methods Engng., 51:791811, 2001.

[14] A. Konyukhov and K. Schweizerhof. Contact formulation via a velocity description allowing efficiency improvements in frictionless contact analysis. Comput. Mech., 33:165-173, 2004.

[15] T.A. Laursen. Formulation and treatment of frictional contact problems using finite elements. Dissertation, SUDAM Report No. 92-6, Stanford University, 1992.

[16] T.A. Laursen. Computational contact and impact mechanics. Springer, 2002.

[17] H. Parisch. A continuum-based shell theory for non-linear applications. Int. J. Numer. Methods Engng., 38:1855-1883, 1995.

[18] X. Qiu, M.E. Plesha, and D.W. Meyer. Stiffness matrix integration rules for contact-friction finite elements. Comput. Methods Appl. Mech. Engng., 93:385399, 1991.

[19] H. Schoop. Oberflächenorientierte Schalentheorien endlicher Verschiebungen. Ingenieur-Archiv, 56:427-437, 1986.

[20] J.C. Simo and F. Armero. Geometrically non-linear enhanced strain mixed methods and the method of incompatible modes. Int. J. Numer. Methods Engng., 33:1413-1449, 1992.

[21] J.C. Simo and T.A. Laursen. An Augmented Lagrangian treatment of contact problems involving friction. Comp. Struct., 42(1):97-116, 1992.

[22] J.C. Simo, R.L. Taylor, and K.S. Pister. Variational and projection methods for volume constraint in finite deformation elasto-plasticity. Comput. Methods Appl. Mech. Engng., 51:177-208, 1985. 
[23] R.L. Taylor and P. Papadopoulos. On a patch test for contact problems in two dimensions. In: Wriggers P., Wagner W. eds. Computational methods in nonlinear mechanics., Springer: Berlin; 1991, p. 690-702.

[24] P. Wriggers. Computational Contact Mechanics. Wiley, 2002.

[25] P. Wriggers, J.C. Simo, and R.L. Taylor. Penalty and Augmented Lagrangian formulations for contact problems. In J. Middleton and G. Pande, editors, Proceedings of NUMETA Conference. Balkema, Roterdam, 1985.

[26] P. Wriggers, V. Vu, and E. Stein. Finite element formulation of large deformation impact-contact problem with friction. Comp. Struct., 37:319-331, 1990.

[27] G. Zavarise, P. Wriggers. A Segment-to-Segment Contact Strategy. Math. Comput. Modeling. Vol. 28, No. 4-8, pp. 497-515, 1998. 